\title{
Rare earth and trace element signatures for assessing an impact of rock mining and processing on the environment: Wiśniówka case study, south-central Poland
}

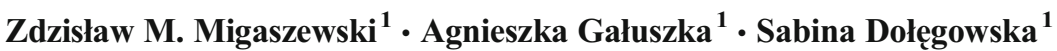

Received: 31 May 2016/Accepted: 14 September 2016/Published online: 25 September 2016

(C) The Author(s) 2016. This article is published with open access at Springerlink.com

\begin{abstract}
A detailed hydrogeochemical study was performed in the Wiśniówka mining area (south-central Poland). This covered three acid pit bodies, historic tailings acid ponds, acid pools, and additionally two neighboring rivers. All these acid mine drainage (AMD) waters are characterized by the $\mathrm{pH}$ in the range of 1.7 (pools) to 3.5 (tailings ponds). The most interesting is the Podwiśniówka acid pit lake that shows a very low $\mathrm{pH}(2.2-2.5)$ and very high concentrations of $\mathrm{SO}_{4}{ }^{2-}$ (2720-5460 mg/L), Fe (545-1140 mg/L), Al (86.2 mg/L), As $(9603-24,883 \mu \mathrm{g} / \mathrm{L})$, Co $(1317-3458 \mu \mathrm{g} / \mathrm{L})$, Cr $(753-$ $2047 \mu \mathrm{g} / \mathrm{L}), \mathrm{Cu}(6307-18,879 \mu \mathrm{g} / \mathrm{L}), \mathrm{Ni}(1168-3127 \mu \mathrm{g} / \mathrm{L})$, and rare earth element (REE) (589-1341 $\mu \mathrm{g} / \mathrm{L})$. In addition, seeps that drain the Podwiśniówka mine tailings and partly aggregate piles form strong acid pools in the mining area. Along with these pools, in which As and REE contents reach 369,726 and $6288 \mu \mathrm{g} / \mathrm{L}$, respectively, these waters are among the most distinctive As- and REE-rich AMD surface waters across the world. It is noteworthy that the Podwiśniówka acid pit lake and Wiśniówka Duża acid pit sump exhibit different element signatures and REE concentration patterns normalized to North American Composite Shale (NASC): the Podwiśniówka acid pit lake always shows a characteristic roof-shaped medium REE (MREE) profile with distinct enrichments in $\mathrm{Gd}, \mathrm{Eu}$, and $\mathrm{Tb}$ whereas the other one displays a step-shaped heavy REE (HREE) profile with positive $\mathrm{Tb}$ and Gd anomalies. The REE undergo fractionation during weathering and the subsequent leaching of dissolved and
\end{abstract}

Responsible editor: Philippe Garrigues

Zdzisław M. Migaszewski

zmig@ujk.edu.pl

1 Jan Kochanowski University, Świętokrzyska 15G, 25-406 Kielce, Poland suspended fractions from rocks to acid water bodies where these and other elements are further fractionated by geochemical processes. This study shows that the individual REE have greater affinities for $\mathrm{Mn}, \mathrm{HREE}$ for $\mathrm{Fe}$ and $\mathrm{SO}_{4}{ }^{2-}$, and only $\mathrm{La}$ and $\mathrm{Ce}$ for Al. This specific water geochemistry has enabled us to (i) pinpoint the location of AMD "hot spots" originated from quartzite mining and processing operations conducted by current and previous mining companies, (ii) predict the directions and effects of future strip mining for quartzites in the Wiśniówka Duża and Podwiśniówka open pits, and (iii) evaluate the potential impact of mining and processing effluents on the quality of rivers.

Keywords REE enrichments $\cdot$ Metal(loid)s $\cdot$ Pyrite $\cdot$ Acid mine drainage $\cdot$ Rock mining impact

\section{Introduction}

The origin, transport and fate of elements, including rare earth elements (REEs) and arsenic, in different acid mine drainage (AMD) sites have been studied for decades (e.g., HudsonEdwards et al. 1999; Johannesson and Zhou 1999; Plumlee et al. 1999; Winterbourn et al. 2000; Worall and Pearson 2001; Druschel et al. 2004; Bednar et al. 2005; Gammons et al. 2005; Olías et al. 2006; Pérez-López et al. 2010; Nordstrom 2011; Grawunder et al. 2014; Migaszewski and Gałuszka 2015). These studies have also included geochemical processes that effect remobilization of trace and minor elements from sulfide ore and coal deposits, mineralized rock formations, and mine wastes to surface and underground waters (e.g., Reichenbach 1994; Monterroso and Macías 1998; Nordstrom 2011). This remobilization is initiated by oxidation of pyrite and ironbearing sulfides in the presence of two natural oxidants, i.e., oxygen and subsequently at a lower $\mathrm{pH}$ by more effective ferric 
$\left(\mathrm{Fe}^{3+}\right)$ iron (e.g., Garrels and Thompson 1960; Moses et al. 1987; Nordstrom and Alpers 1999a). This process is a potential source of acidification and dispersal of hazardous metal(loid)s into waters, sediments, soils, and biota (e.g., Aguilar et al. 2004; Younger et al. 2005; Cánovas et al. 2008; Martínez-Martínez et al. 2013). Moreover, this triggers a chain of reactions that lead to formation of secondary minerals (e.g., schwertmannite, ferrihydrite, goethite, lepidocrocite, hematite, hydroxy-green rusts, jarosite) that seem to play a decisive role in controlling the $\mathrm{pH}$ of pit pond or lake waters (e.g., Bingham et al.1990, 1992; Schwertmann et al. 1995; Acero et al. 2006). These complex processes induced primarily by anthropogenic activity are termed acid mine drainage. Because AMD waters jeopardize health of abiotic and biotic systems, the studies have also encompassed specific geochemical signatures to assess a metal(loid) pollution extent (Migaszewski et al. 2015).

The REEs have found an application in fingerprinting geologic and anthropogenic sources and in investigating geochemical processes that lead to fractionation of this element group in various environmental compartments, but particularly in surface and underground waters (e.g., Johannesson and Lyons 1995; Verplanck et al. 2004; Bozau et al. 2004; Gammons et al. 2005; Knappe et al. 2005; Leybourne and Johannesson 2008; Kulaksiz and Bau 2011; Migaszewski and Gałuszka 2015, 2016). In the surface water systems, the REE undergo fractionation between colloid/suspended matter and dissolved fractions (Ingri et al. 2000). The mobile REE fractions are very sensitive to small variations in the $\mathrm{pH}$, redox potential, salinity, and concentrations of chelating agents, and participate in water-solid sorption-desorption, solution, and surface complexation involving inorganic and organic ligands (e.g., $\mathrm{CO}_{3}{ }^{2-}, \mathrm{PO}_{4}{ }^{3-}$, humic and fulvic acids), and coprecipitation with colloids and suspended matter. Of particular interest are acid AMD streams, ponds, and lakes that provide a direct insight into local and regional geologic sources highlighted by a number of specific REE profiles (e.g., Bozau et al. 2004; Verplanck et al. 2004; Grawunder et al. 2014; Migaszewski et al. 2015). The AMD waters commonly exhibit high concentrations of REE in the range of tens to hundreds micrograms per liter (Verplanck et al. 2001; Protano and Riccobono 2002; Gammons et al. 2005; Borrego et al. 2012), and different metal(loid)s, especially $\mathrm{Fe}$ and As. The mentioned last reflects the fate of other trace elements in AMD aqueous environments. Arsenic undergoes geochemical interactions within the water-colloid-ochreous precipitate system. Weathering of pyrite releases As which is trapped by unstable schwertmannite. The transformation of this mineral into ferrihydrite at the $\mathrm{pH}$ of 2.5-4.5 and subsequently into goethite at the $\mathrm{pH}$ of 3-7 leads to desorption of As. The decrease in the $\mathrm{pH}$ brings about reductive dissolution of iron oxyhydroxides triggering also the release of As (e.g., Bingham et al. 1990, 1992; Smedley and Kinniburgh 2002; Bednar et al. 2005; Courtin-Nomade et al. 2005).
The previous studies conducted during 2006-2012 encompassed only two water bodies, i.e., the existing Wiśniówka Mała acid pit lake (Fig. 1) and the former Podwiśniówka acid pit pond. Their purpose was to identify geochemical processes of AMD water generation based on determinations of $\delta^{34} \mathrm{~S}-\mathrm{FeS}_{2}, \delta^{34} \mathrm{~S}_{-} \mathrm{SO}_{4}{ }^{2-}, \delta^{18} \mathrm{O}-\mathrm{SO}_{4}{ }^{2-}$, $\delta^{18} \mathrm{O}-\mathrm{H}_{2} \mathrm{O}$, and $\delta \mathrm{D}-\mathrm{H}_{2} \mathrm{O}$ and selected trace metal(loid)s (Migaszewski et al. 2007a, 2008, 2009, 2013, 2014). In addition, the scope of investigation also covered mineralogical and petrological examinations of pyrite mineralization zone exposed on the western wall of the "Podwiśniówka" quarry (Migaszewski et al. 2007b; Migaszewski and Gałuszka 2010). The situation changed when the new mining company started intensive quartzite extraction in the Podwiśniówka quarry. This operation lasted from the spring to the fall of 2014. Deep quarrying for quartzites along the pyrite-bearing zone has induced generation of AMD waters, leaving an acid pit lake on the lowest mining bench (Fig. 2a). This water shows totally different chemistry highlighted by much higher levels of sulfates, arsenic, and trace metals compared to those recorded in the former shallow acid pit pond. To make this picture worse, formation of strongly As- and metalconcentrated acid pools near tailings piles and the use of water from the abandoned Wiśniówka Mała pit lake for washing of crushed quartzite aggregate from the Podwiśniówka quarry have posed an additional potential threat to the environment.

The principal objectives of the study presented here are to (i) determine the current hydrogeochemistry of AMD water bodies, (ii) apply characteristic element profiles to pinpoint rock mining and processing operations, and (iii) evaluate AMD water influence on the neighboring surface water systems.

\section{Materials and methods}

\section{Study area location and geologic framework}

The present study covered a large Wiśniówka mining area located about $5 \mathrm{~km}$ north of Kielce, the capital of the Holy Cross Mountains region (south-central Poland). The study area lies within a dismembered massif (elevation of $455.7 \mathrm{~m}$ above sea level (a.s.l.)) assigned to the western part of the Main Range of the Holy Cross Mountains. The Wiśniówka Massif is characterized by highly complicated tectonics with intense folding and faulting and very complex stratigraphy, being the subject of controversy and dispute among geologists for decades (Żylińska et al. 2006; Konon 2008, and references therein). There are three quarries occurring in the mining area (from the west to the east): Wiśniówka Mała, Wiśniówka Duża, and Podwiśniówka (Fig. 1). The basic lithostratigraphic unit of the Wiśniówka Massif is the Wiśniówka Sandstone Formation (Orłowski 1975) that consists of thick-bedded 
Fig. 1 Location of study area with sampling sites.

Explanations: 1, Podwiśniówka lake; 2, Wiśniówka Duża pit sump pond; 3 , Wiśniówka Mała lake; 4 , historic tailings pile ponds; 5 , Wiśniówka Duża pool; 6, Wiśniówka aggregate processing plant pool; 7 Wiśniówka aggregate processing plant pool; 8, Lubrzanka River upstream; 9, Lubrzanka River downstream; 10, Silnica River upstream; 11, Silnica River downstream; 12, culvert under highway E-73; 13, culvert under housing development; 14, culvert under railroad track in Kajetanów

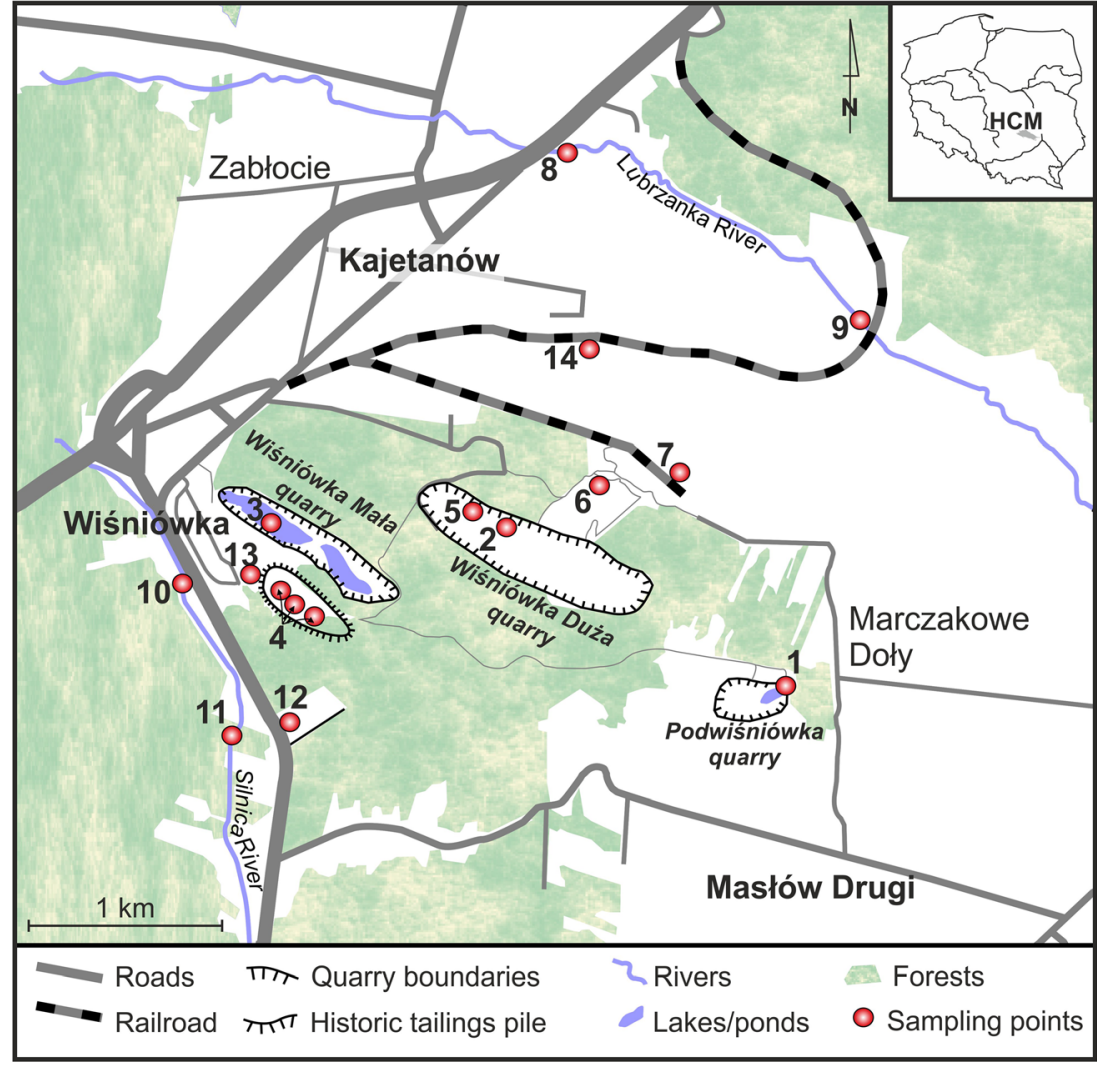

Upper Cambrian (Furongian) quartzites and quartzitic sandstones (fine-grained quartz arenites) interbedded with quartzitic siltstones, clayey shales and subordinate bentonites and tuffites, in places with pyrite mineralization zones (Jaworowski and Sikorska 2006).
These pyritiferous zones, which are fully exposed only in the Podwiśniówka and partly in the Wiśniówka Duża quarries, are a source of AMD water generation. The Podwiśniówka quarry is distinguished by specific mineralogical features of predominant crypto- to micro-grained As-rich pyrite (gel-
Fig. 2 A general view of a Podwiśniówka acid pit lake, $\mathbf{b}$ Wiśniówka Duża pit sump, c Wiśniówka Mała acid pit lake, d historic tailings acid pond

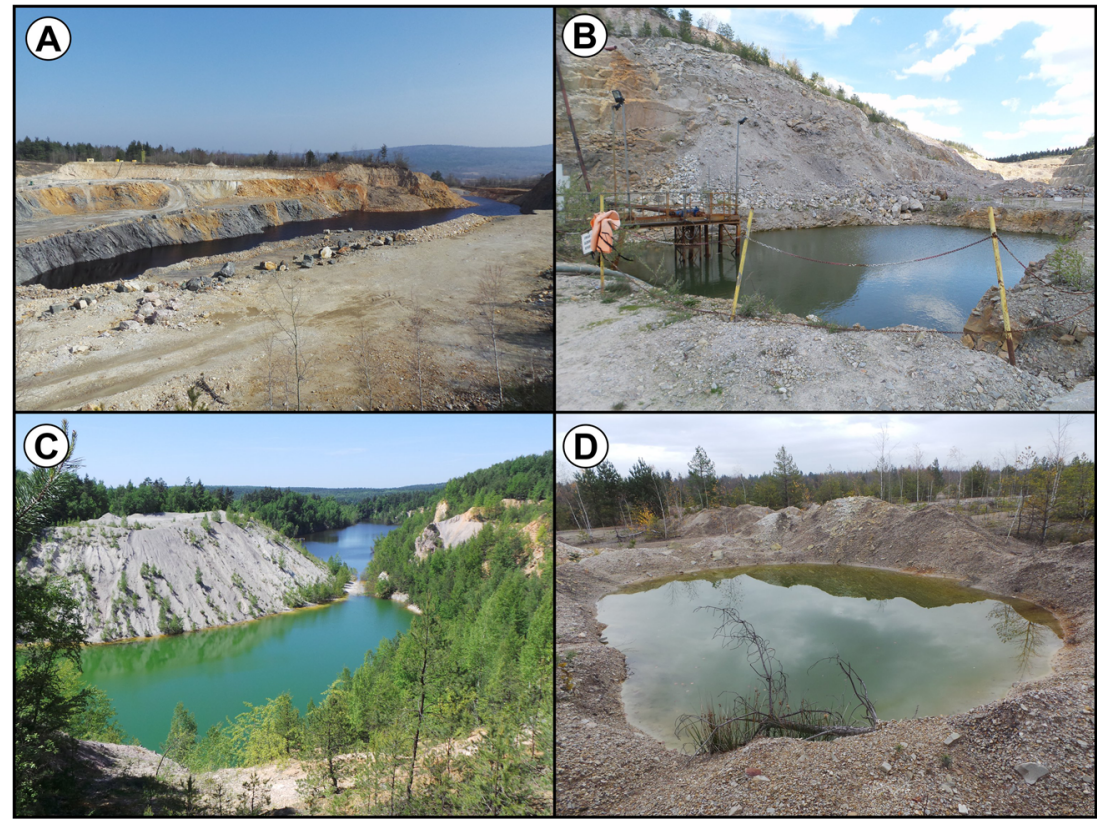


pyrite) that favor its rapid oxidation as evidenced by the previous studies (Migaszewski et al. 2007a, 2008). Moreover, in the northern part of this quarry, hematite and goethite mineralization zone crops out at the surface. There are xenoliths of pyritiferous quartzites within this iron(III) oxide and oxyhydroxide zone. It is noteworthy that hydrothermal REEbearing minerals (gorceixite, florencite, xenotime) occur primarily within clayey shale and subordinate tuffite interbeds of the Podwiśniówka quarry as evidenced by the previous studies (Migaszewski et al. 2007b; Migaszewski and Gałuszka 2010).

Consequently, there are numerous acid water bodies in the mining area showing different chemistry. These include Podwiśniówka acid pit lake, Wiśniówka Mała acid pit lake, Wiśniówka Duża pit sump, small ponds on the surface of historical tailings piles, and periodic highly acidic and metal(loid) concentrated pools that drain mine and processing tailings piles or exposed pyrite-rich zones where the landscape was stripped for mining (Fig. 2a-d). Of all the examined acid water bodies, the most interesting is the Podwiśniówka acid pit lake that takes up an area of over 1 ha with an average depth of about $2.3 \mathrm{~m}$ (Figs. 1 and 2a). Its water level coordinate is approx. $399.0 \mathrm{~m}$ a.s.1., and its cubature is about $23,600 \mathrm{~m}^{3}$. The Wiśniówka Duża pit sump has in turn an area of about $260 \mathrm{~m}^{2}$ and a depth of about $5.4 \mathrm{~m}$ (Figs. 1 and 2b) whereas Wiśniówka Mała pit lake covers an area of about 5.2 ha with an average depth of about $11 \mathrm{~m}$ (Figs. 1 and 2c). The coordinates of their water levels are 367.7 and $355.7 \mathrm{~m}$ a.s.l., respectively. During over 100 years of intermittent quarrying, pyrite-bearing waste material was stockpiled on a few hectares of land close to the southwestern boundary of Wiśniówka mining area (no. 4 in Fig. 1). The mining area borders on two rivers: Lubrzanka to the north and Silnica to the west (Fig. 1).

\section{Field geochemical measurements and sampling}

Fieldwork was conducted from July 14 of 2014 through March 30 of 2016. This included sampling of AMD waters (35 samples) and additionally river waters (15 samples) for chemical analysis. Sampling, which is a crucial stage of each environmental study, was carried out according to US EPA recommendations (ISO/IEC:17025: 2005; NORDIC INNOVATION CENTRE 2007). All the water samples for trace element measurements were filtered through $0.45-\mu \mathrm{m}$ pore-sized polytetrafluoroethylene (PTFE) syringe filters and placed in 50-mL polypropylene vials. The filtered and untreated water samples were transported to the Geochemical Laboratory of the Institute of Chemistry, Jan Kochanowski University, in Kielce on the same day and stored in a refrigerator at a temperature of about $4-6{ }^{\circ} \mathrm{C}$.

Fieldwork also included direct measurements of $\mathrm{pH}$, electric conductivity (EC), total dissolved solids (TDS), and temperature $(\mathrm{T})$ of water, using a manual microprocessor $\mathrm{pH} /$ Eh-meter SP300 with a combination $\mathrm{pH}$-electrode probe Winlab and a manual microprocessor EC-meter SC300 with a four-electrode combined conductivity probe WinLab (both Slandi, Poland) equipped with temperature sensors. In addition, alkalinity $\left(\mathrm{CaCO}_{3} \mathrm{mg} / \mathrm{L}\right)$, and concentrations of $\mathrm{SO}_{4}{ }^{2-}$, $\mathrm{PO}_{4}{ }^{3-}$, and total $\mathrm{Fe}$ were determined on-site using a field spectrophotometers LF-205 and LF-300 Slandi, Poland.

During sample collection, transport, storage, and preparation, procedures were followed to minimize the possibility of contamination. A set of water samples included one blank (deionized water from the laboratory that was processed in the field along with the environmental samples) and one replicate sample for each sampling series.

\section{Sample preparation and chemical analysis}

All the samples were analyzed for $\mathrm{Al}, \mathrm{As}, \mathrm{Cd}, \mathrm{Co}, \mathrm{Cr}, \mathrm{Cu}, \mathrm{Mn}$, $\mathrm{Ni}, \mathrm{Pb}, \mathrm{Tl}$, and $\mathrm{Zn}$, using an Inductively Coupled PlasmaQuadropole Mass Spectrometer (ICP-QMS; model ELAN DRC II, Perkin Elmer). Operating conditions were as follows: sweeps/reading, 20; readings/replicate, 3; replicates, 4; nebulizer gas flow, $0.99 \mathrm{~L} / \mathrm{min}$; plasma gas flow, $1.5 \mathrm{~L} / \mathrm{min}$; lens voltage, 6.50-6.75 V; plasma power, $1275 \mathrm{~W}$. The ICP-QMS instrument was optimized with a standard daily procedure. For trace element determinations, a set of Multielement Calibration Standards Perkin Elmer solutions were applied. Quantification of REE was done with an external standard method. Working solutions with REE concentrations in the range of $0.1-10 \mu \mathrm{g} / \mathrm{L}$ were used to construct the four-point calibration curve $\left(r^{2}>0.999\right)$. These solutions were prepared using the multielement standard solution from PerkinElmer (10 mg/L REE in $2 \%$ nitric acid). Dilution of standards and samples was done with $2 \%$ nitric acid prepared from ultrapure $\mathrm{HNO}_{3}$ (65\%, Merck) and distilled water. Iridium at a concentration of $100 \mu \mathrm{g} / \mathrm{L}$ (prepared from Perkin-Elmer single-element standard solution containing $1000 \mathrm{mg} / \mathrm{L} \mathrm{Ir}$ ) was applied as an internal standard. Physical interferences were eliminated by the use of internal standard and sample dilution (10-100 times, depending on concentrations of total dissolved solids). Spectral interferences were eliminated by application of the following correction equations:

$$
\begin{aligned}
{ }^{142} \mathrm{Nd}:{ }^{142} \mathrm{Nd} & =-0.125653 \times{ }^{140} \mathrm{Ce} \\
{ }^{149} \mathrm{Sm}:{ }^{149} \mathrm{Sm} & =-0.012780 \times{ }^{157} \mathrm{Gd} \\
{ }^{160} \mathrm{Gd}:{ }^{160} \mathrm{Gd} & =-0.004016 \times{ }^{163} \mathrm{Dy} \\
{ }^{164} \mathrm{Dy}:{ }^{164} \mathrm{Dy} & =-0.047902 \times{ }^{166} \mathrm{Er} \\
{ }^{172} \mathrm{Yb}:{ }^{172} \mathrm{Yb} & =-0.005865 \times{ }^{178} \mathrm{Hf}
\end{aligned}
$$

The standard reference materials (SRMs) used for measuring element concentrations in the samples were NIST $1643 \mathrm{e}$ (trace elements in water) and the geologic multielement 
reference material (GM-ERM) PPREE1 (Table 2 in Verplanck et al. (2001)) for waters. Quality control included both accuracy (SRM) and precision (triplicates). The average recovery of elements from the SRM was 80-110\%, and the uncertainty of the method was below $10 \%$. The relative standard deviation (RSD) values were $<4 \%$ for most of the analyzed samples.

\section{REE ratios and enrichments}

For the purpose of this study, the REEs (= lanthanides) were divided into two conventionally termed subgroups: (i) light REE (LREE) including La through Eu and (ii) heavy REE (HREE) from Gd through Lu, respectively. The third discriminated subgroup, medium REE (MREE; Sm through Ho) partly overlaps LREE and HREE. In order to compare REE concentrations in different water bodies of the Wiśniówka mining area, the results derived from these element measurements were normalized to North American Shale Composite (NASC) (Haskin et al. 1968; Gromet et al. 1984). This eliminates the characteristic "zigzag" REE distribution patterns and enables us to identify any individual REE anomalies in various geologic and environmental materials (Migaszewski and Gałuszka 2015).

The $\mathrm{Ce} / \mathrm{Ce}_{\mathrm{NASC}}{ }^{*}$ ratio in the Wiśniówka AMD waters was calculated from the Eq. (1) (Bau and Dulski 1996) and reported in Table 4:

$\mathrm{Ce} / \mathrm{Ce}_{\mathrm{NASC}}{ }^{*}=\mathrm{Ce}_{\mathrm{NASC}} /\left(0.5 \mathrm{La}_{\mathrm{NASC}}+0.5 \operatorname{Pr}_{\mathrm{NASC}}\right)$

where $\mathrm{Ce}_{\mathrm{NASC}}{ }^{*}$ is a background concentration whereas $\mathrm{La}_{\mathrm{NASC}}$ and $\operatorname{Pr}_{\mathrm{NASC}}$ are the NASC-normalized La and Pr concentrations, respectively.

The same equation structure was consistently employed for $\mathrm{Eu} / \mathrm{Eu}_{\mathrm{NASC}}{ }^{*}, \mathrm{Gd} / \mathrm{Gd}_{\mathrm{NASC}}{ }^{*}$, and $\mathrm{Tb} /$ $\mathrm{Tb}_{\text {NASC }}{ }^{*}$ ratios. However, due to distinct enrichment of $\mathrm{Gd}$, it was inadmissible to use this element in calculation of Eu anomaly. This is the reason why modification of equation had to be made ( $\mathrm{Gd}$ was substituted for Dy). The $\mathrm{Eu} / \mathrm{Eu}_{\mathrm{NASC}}{ }^{*}$ ratio in all the examined acid waters was calculated from the Eq. (2) (German et al. 1991, modified by the present authors) and given in Table 4:

$\left.\mathrm{Eu} / \mathrm{Eu}_{\mathrm{NASC}}{ }^{*}=\mathrm{Eu}_{\mathrm{NASC}}\right) /\left(\left(\mathrm{Sm}_{\mathrm{NASC}}+\mathrm{Dy}_{\mathrm{NASC}}\right) / 2\right)$

Similarly, because of distinct enrichment of $\mathrm{Tb}$, this element was not used in calculation of Gd anomaly. The $\mathrm{Gd} /$ $\mathrm{Gd}_{\text {NASC }}{ }^{*}$ ratio was computed from the Eq. (3) and presented in Table 4:

$\mathrm{Gd} / \operatorname{Gd}_{\mathrm{NASC}}{ }^{*}=\operatorname{Gd}_{\mathrm{NASC}} /\left(\left(\mathrm{Sm}_{\mathrm{NASC}}+\mathrm{Dy}_{\mathrm{NASC}}\right) / 2\right)$
$\mathrm{The} \mathrm{Tb} / \mathrm{Tb}_{\mathrm{NASC}}{ }^{*}$ ratio was in turn computed from Eq. (4) and presented in Table 4:

$\left.\mathrm{Tb} / \mathrm{Tb}_{\mathrm{NASC}}{ }^{*}=\mathrm{Tb}_{\mathrm{NASC}}\right) /\left(\left(\mathrm{Sm}_{\mathrm{NASC}}+\mathrm{Dy}_{\mathrm{NASC}}\right) / 2\right)$

where $\mathrm{Eu} / \mathrm{Gd} / \mathrm{Tb}_{\mathrm{NASC}}{ }^{*}$ are background concentrations where-

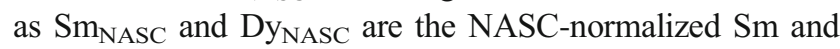
Dy concentrations, respectively.

Values below 0.8 are indicative of negative anomalies whereas those above 1.2 point to positive anomalies (Grawunder et al. 2014). In addition, the La $\mathrm{N}_{\mathrm{NASC}} / \mathrm{Yb}_{\mathrm{NASC}}$ and $\mathrm{Sm}_{\mathrm{NASC}} / \mathrm{Yb}_{\mathrm{NASC}}$ ratios were computed to assess depletion $(<0.8)$ or enrichment $(>1.2)$ of distinguished REE subgroups: LREE (La), MREE (Sm), and HREE (Yb).

\section{Statistical analysis}

Data processing included the Microsoft Excel application for summary statistics and graphical presentation of REE patterns, and cluster and factor analysis. In the statistics calculations, the censored (below lower limit of determination) values were replaced with arbitrary values equal to $50 \%$ of their determination limit. Both cluster and factor analysis is a multivariate analysis. The former enables us to form groups of related variables. Based on similarities and dissimilarities within and between classes, the objects are divided into "clusters." The data obtained were normalized and standardized using STATISTICA Base software (StatSoft Inc.), with the linkage distances for a particular case divided by the maximal linkage distance $\left(\mathrm{D}_{\text {link }} / \mathrm{D}_{\max }\right)$. The data derived from this study were originally centered by Box-Cox function with all variables standardized later. The cluster analysis was done using the Ward's method with a square Euclidean distance as a measure of similarity. The Ward's method enables an analysis of variance approach to evaluate the distances between the clusters in order to minimize the sum of squares of any two clusters (that can be formed at each step).

The factor analysis in turn allows us to describe variability among the dataset. This groups similar variables into specific structures without distinguishing between dependent and independent variables. In the present study, two main factors were retained (explained variance: F1 14.01; F2 12.94). The first factor is loaded by $\mathrm{pH}, \mathrm{EC}, \mathrm{As}, \mathrm{Co}, \mathrm{Cu}, \mathrm{Fe}, \mathrm{Ni}, \mathrm{Sc}$, and $\mathrm{SO}_{4}{ }^{2-}$ and the other by $\mathrm{Al}, \mathrm{Ce}, \mathrm{Cd}, \mathrm{La}, \mathrm{Nd}, \mathrm{Pr}$, and $\mathrm{Zn}$. With regard to other variables, cross-loading is observed. The scatterplot (not included for the sake of brevity) obtained does not depict the independent factors.

\section{Petrographic study}

The principal objective of the preliminary petrographic study was to find "signatures" in rock-water interactions. The ore 
Table 1 The mean values of measured physicochemical and chemical parameters in the Wiśniówka AMD water bodies

\begin{tabular}{|c|c|c|c|c|c|}
\hline Parameter & $\begin{array}{l}\text { Podwiśniówka } \\
\text { pit lake }(N=6)\end{array}$ & $\begin{array}{l}\text { Wiśniówka } \\
\text { Duża pit sump } \\
(N=5)\end{array}$ & $\begin{array}{l}\text { Wiśniówka } \\
\text { Mała pit lake } \\
(N=5)\end{array}$ & $\begin{array}{l}\text { Historic tailings } \\
\text { pile ponds } \\
(N=6)\end{array}$ & $\begin{array}{l}\text { Maximum } \\
\text { allowable limits } \\
\text { MEP (2014) }\end{array}$ \\
\hline $\mathrm{pH}$ & $2.2-2.5$ & $3.0-3.4$ & $3.0-3.1$ & $3.1-3.5$ & $6.5-9.0$ \\
\hline $\mathrm{Eh}(\mathrm{mV})$ & $266 \pm 6$ & $222 \pm 11$ & $232 \pm 4$ & $211 \pm 12$ & n.d. \\
\hline $\begin{array}{c}\mathrm{EC}(\mathrm{mS} / \\
\mathrm{cm})\end{array}$ & $5.57 \pm 0.57$ & $1.15 \pm 0.12$ & $1.10 \pm 0.14$ & $1.08 \pm 0.45$ & n.d. \\
\hline $\begin{array}{l}\text { TDS (mg/ } \\
\mathrm{L})\end{array}$ & $2790 \pm 288$ & $574 \pm 63$ & $528 \pm 60$ & $542 \pm 221$ & n.d. \\
\hline $\begin{array}{l}\mathrm{SO}_{4}{ }^{2-} \\
(\mathrm{mg} / \mathrm{L})\end{array}$ & $3978 \pm 885$ & $575 \pm 184$ & $534 \pm 64$ & $608 \pm 360$ & 500 \\
\hline $\begin{array}{l}\mathrm{PO}_{4}^{3-} \\
\quad(\mathrm{mg} / \mathrm{L})\end{array}$ & $7.92 \pm 0.63$ & 0.04 & 0.17 & $0.01 \pm 0.008$ & $\mathrm{n} / \mathrm{a}$ \\
\hline $\begin{array}{l}\text { Alkal. } \\
\text { (mg/L) }\end{array}$ & $75 \pm 23$ & $119 \pm 70$ & $65 \pm 40$ & $88 \pm 68$ & $\mathrm{n} / \mathrm{a}$ \\
\hline $\begin{array}{l}\mathrm{Fe}_{\text {total }} \\
\quad(\mathrm{mg} / \mathrm{L})\end{array}$ & $860 \pm 260$ & $9.1 \pm 4.4$ & $23.3 \pm 10.0$ & $5.2 \pm 4.4$ & 10 \\
\hline $\mathrm{Al}(\mathrm{mg} / \mathrm{L})$ & 86.02 & 18.9 & 25.5 & $10.1 \pm 10.0$ & 3 \\
\hline As $(\mu \mathrm{g} / \mathrm{L})$ & $15,416 \pm 5197$ & $15.1 \pm 11.4$ & $82.4 \pm 52.7$ & $47.5 \pm 38.7$ & 100 \\
\hline $\begin{array}{l}\mathrm{Cd} \\
\qquad(\mu \mathrm{g} / \mathrm{L})\end{array}$ & $3.80 \pm 0.94$ & $0.52 \pm 0.13$ & $0.68 \pm 0.10$ & $0.77 \pm 0.32$ & $400(200)^{\mathrm{a}}$ \\
\hline $\begin{array}{l}\mathrm{Co} \\
\quad(\mu \mathrm{g} / \mathrm{L})\end{array}$ & $2413 \pm 776$ & $200 \pm 66$ & $135 \pm 24$ & $135 \pm 64$ & 1000 \\
\hline $\mathrm{Cr}(\mu \mathrm{g} / \mathrm{L})$ & $1377 \pm 413$ & $41 \pm 20$ & $76 \pm 14$ & $61.8 \pm 60.6$ & 500 \\
\hline $\begin{array}{l}\mathrm{Cu} \\
(\mu \mathrm{g} / \mathrm{L})\end{array}$ & $14,314 \pm 5278$ & $1923 \pm 619$ & $451 \pm 111$ & $446 \pm 348$ & 500 \\
\hline $\begin{array}{l}\mathrm{Mn} \\
\quad(\mu \mathrm{g} / \mathrm{L})\end{array}$ & $1922 \pm 1349$ & $1064 \pm 536$ & $6317 \pm 1606$ & $1871 \pm 343$ & n.d. \\
\hline $\mathrm{Ni}(\mu \mathrm{g} / \mathrm{L})$ & $2303 \pm 783$ & $207 \pm 75$ & $188 \pm 5$ & $243 \pm 123$ & 500 \\
\hline $\mathrm{Pb}(\mu \mathrm{g} / \mathrm{L})$ & $4.86 \pm 3.91$ & $0.68 \pm 1.12$ & $1.01 \pm 1.32$ & $0.91 \pm 0.96$ & 500 \\
\hline $\mathrm{Tl}(\mu \mathrm{g} / \mathrm{L})$ & $7.63 \pm 2.10$ & $0.72 \pm 0.40$ & $0.38 \pm 0.10$ & $0.08 \pm 0.02$ & 1000 \\
\hline $\mathrm{Zn}(\mu \mathrm{g} / \mathrm{L})$ & $419 \pm 293$ & $213 \pm 163$ & $142 \pm 30$ & $215 \pm 33$ & 2000 \\
\hline $\mathrm{Sc}(\mu \mathrm{g} / \mathrm{L})$ & $310 \pm 94$ & $44.6 \pm 22.5$ & $14.2 \pm 5.3$ & $19.0 \pm 25.3$ & $\mathrm{n} / \mathrm{a}$ \\
\hline $\mathrm{Y}(\mu \mathrm{g} / \mathrm{L})$ & $266 \pm 74$ & $109.2 \pm 13.3$ & $40.1 \pm 18.1$ & $111.3 \pm 78.7$ & $\mathrm{n} / \mathrm{a}$ \\
\hline $\mathrm{La}(\mu \mathrm{g} / \mathrm{L})$ & $82 \pm 23$ & $11.40 \pm 2.96$ & $9.43 \pm 2.74$ & $13.6 \pm 7.5$ & $\mathrm{n} / \mathrm{a}$ \\
\hline $\mathrm{Ce}(\mu \mathrm{g} / \mathrm{L})$ & $260 \pm 80$ & $35.9 \pm 9.9$ & $25.2 \pm 8.1$ & $49.5 \pm 30.8$ & $\mathrm{n} / \mathrm{a}$ \\
\hline $\operatorname{Pr}(\mu \mathrm{g} / \mathrm{L})$ & $41 \pm 12$ & $6.18 \pm 1.90$ & $3.69 \pm 1.29$ & $8.7 \pm 6.1$ & $\mathrm{n} / \mathrm{a}$ \\
\hline $\begin{array}{l}\mathrm{Nd} \\
\qquad(\mu \mathrm{g} / \mathrm{L})\end{array}$ & $216 \pm 62$ & $34.6 \pm 9.8$ & $17.9 \pm 6.6$ & $48.1 \pm 34.9$ & $\mathrm{n} / \mathrm{a}$ \\
\hline $\mathrm{Sm}_{(\mu \mathrm{g} / \mathrm{L})}$ & $92 \pm 29$ & $12.76 \pm 3.90$ & $7.19 \pm 2.64$ & $16.03 \pm 11.93$ & $\mathrm{n} / \mathrm{a}$ \\
\hline $\mathrm{Eu}(\mu \mathrm{g} / \mathrm{L})$ & $24.5 \pm 6.7$ & $3.19 \pm 1.13$ & $1.94 \pm 0.86$ & $4.26 \pm 3.13$ & $\mathrm{n} / \mathrm{a}$ \\
\hline $\begin{array}{l}\mathrm{Gd} \\
\quad(\mu \mathrm{g} / \mathrm{L})\end{array}$ & $113.3 \pm 29.2$ & $20.3 \pm 6.4$ & $10.5 \pm 4.0$ & $23.8 \pm 17.6$ & $\mathrm{n} / \mathrm{a}$ \\
\hline $\mathrm{Tb}(\mu \mathrm{g} / \mathrm{L})$ & $15.6 \pm 4.1$ & $3.28 \pm 1.03$ & $1.57 \pm 0.69$ & $3.80 \pm 2.71$ & $\mathrm{n} / \mathrm{a}$ \\
\hline $\begin{array}{l}\text { Dy } \\
\qquad(\mu \mathrm{g} / \mathrm{L})\end{array}$ & $74.0 \pm 19.2$ & $19.4 \pm 6.3$ & $8.25 \pm 3.82$ & $20.9 \pm 15.3$ & $\mathrm{n} / \mathrm{a}$ \\
\hline $\begin{array}{l}\text { Ho } \\
\quad(\mu \mathrm{g} / \mathrm{L})\end{array}$ & $12.5 \pm 3.2$ & $3.85 \pm 1.24$ & $1.49 \pm 0.71$ & $3.96 \pm 2.87$ & $\mathrm{n} / \mathrm{a}$ \\
\hline $\operatorname{Er}(\mu \mathrm{g} / \mathrm{L})$ & $30.6 \pm 7.9$ & $10.68 \pm 2.91$ & $3.80 \pm 1.78$ & $10.4 \pm 7.6$ & $\mathrm{n} / \mathrm{a}$ \\
\hline $\operatorname{Tm}_{(\mu \mathrm{g} / \mathrm{L})}$ & $3.74 \pm 1.10$ & $1.50 \pm 0.38$ & $0.49 \pm 0.25$ & $1.29 \pm 0.94$ & $\mathrm{n} / \mathrm{a}$ \\
\hline $\begin{array}{l}\mathrm{Yb} \\
\quad(\mu \mathrm{g} / \mathrm{L})\end{array}$ & $24.40 \pm 6.14$ & $9.31 \pm 2.39$ & $2.98 \pm 1.62$ & $8.06 \pm 6.08$ & $\mathrm{n} / \mathrm{a}$ \\
\hline $\mathrm{Lu}(\mu \mathrm{g} / \mathrm{L})$ & $3.54 \pm 1.01$ & $1.37 \pm 0.40$ & $0.44 \pm 0.25$ & $1.16 \pm 0.89$ & $\mathrm{n} / \mathrm{a}$ \\
\hline $\begin{array}{l}\sum \mathrm{REE} \\
(\mu \mathrm{g} / \mathrm{L})\end{array}$ & $992.88 \pm 281.99$ & $173.67 \pm 50.07$ & $94.85 \pm 35.19$ & $213.57 \pm 147.41$ & \\
\hline $\begin{array}{l}\text { LREE } \\
\qquad(\mu \mathrm{g} / \mathrm{L})\end{array}$ & $715.26 \pm 211.18$ & $103.95 \pm 29.36$ & $63.31 \pm 22.18$ & $140.21 \pm 94.18$ & \\
\hline
\end{tabular}


Table 1 (continued)

\begin{tabular}{llllll}
\hline Parameter & $\begin{array}{l}\text { Podwiśniówka } \\
\text { pit lake }(N=6)\end{array}$ & $\begin{array}{l}\text { Wiśniówka } \\
\text { Duża pit sump } \\
(N=5)\end{array}$ & $\begin{array}{l}\text { Wiśniówka } \\
\text { Mała pit lake } \\
(N=5)\end{array}$ & $\begin{array}{l}\text { Historic tailings } \\
\text { pile ponds } \\
(N=6)\end{array}$ & $\begin{array}{l}\text { Maximum } \\
\text { allowable limits } \\
\text { MEP }(2014)\end{array}$ \\
\hline $\begin{array}{c}\mathrm{HREE} \\
(\mu \mathrm{g} / \mathrm{L})\end{array}$ & $277.62 \pm 71.64$ & $69.72 \pm 20.91$ & $29.54 \pm 13.12$ & $73.37 \pm 53.79$ & \\
$\begin{array}{c}\mathrm{MREE} \\
(\mu \mathrm{g} / \mathrm{L})\end{array}$ & $331.95 \pm 91.03$ & $55.55 \pm 31.30$ & $22.72 \pm 7.02$ & $72.74 \pm 53.26$ & \\
\hline
\end{tabular}

Element and sulfate concentrations, and $\mathrm{pH}$ values exceeding maximum allowable limits (acc, to the Regulation of the Ministry of Environmental Protection, Poland, 2014) are italicized

Alkal. alkalinity $\left(\mathrm{CaCO}_{3} \mathrm{mg} / \mathrm{L}\right)$, n.d. not determined, $n / a$ not applicable

${ }^{a}$ Daily (monthly) discharge

and gangue minerals and rocks of the Wiśniówka Duża and additionally Podwiśniówka quarries were examined using stereoscopic and petrographic microscopes (Leica M205 A, Nikon LV 100 Pol with CITL Cathodoluminescence module MK5-2) and a scanning electron microscope (SEM) LEO
1430 (signal $\mathrm{A}=\mathrm{SEI}$, and BEI, magnification $=\times 1310$ 1850 , EHT $=15.00 \mathrm{kV}, \mathrm{WD}=16-19 \mathrm{~mm})$. Semiquantitative chemical analysis of pyrite was done with an EDS ISIS Detector (Oxford Instruments Ltd.). For this purpose, two standards, i.e., 30. Pyrite and 19. Skutterudite (SPI

Table 2 The $\mathrm{pH}$ values and concentrations of REE, sulfates, and selected trace elements in acid water bodies of the Wiśniówka mining area vs. other AMD sites showing high As and REE concentrations

\begin{tabular}{|c|c|c|c|c|c|c|c|c|c|}
\hline Localization & $\mathrm{pH}$ & $\mathrm{SO}_{4}^{2-}$ & $\mathrm{Fe}_{\text {total }}$ & $\sum \mathrm{REE}$ & As & $\mathrm{Co}$ & $\mathrm{Cr}$ & $\mathrm{Cu}$ & $\mathrm{Ni}$ \\
\hline & & $\mathrm{mg} / \mathrm{L}$ & $\mu \mathrm{g} / \mathrm{L}$ & & & & & & \\
\hline 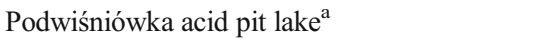 & 2.4 & 3978 & 860 & 993 & 15,416 & 2413 & 1377 & 14,314 & 2303 \\
\hline Wiśniówka Duża quarry (acid pool no. 5) & 1.8 & 36,250 & 5800 & 2433 & 369,726 & 1814 & 2321 & 8742 & 4768 \\
\hline Wiśniówka processing plant (acid pool no. 6) & 2.8 & 91,800 & 950 & 6288 & 16,721 & 2776 & 1349 & 6090 & 2936 \\
\hline Wiśniówka processing plant (acid pool no. 7) & 1.7 & 26,950 & 6572 & 4306 & 288,850 & 5100 & 15,850 & 19,000 & 9475 \\
\hline Serwis acid pool P1/1, Poland ${ }^{\mathrm{b}}$ & 2.6 & n.d. & 4.6 & 1066 & $<0.01$ & 700 & 10 & 0.2 & 200 \\
\hline Richmond Mine, Iron Mountain, $\mathrm{USA}^{\mathrm{c}}$ & n.d. & n.d. & 68,100 & n.d. & 850,000 & 3.6 & 2.6 & 9800 & 6.3 \\
\hline Tailings Stock, Carnoulés, France ${ }^{\mathrm{d}}$ & $\sim 1.2$ & 17,831 & 4355 & n.d. & 638,000 & n.d. & n.d. & n.d. & n.d. \\
\hline Reigous Creek, Carnoulés, France ${ }^{\mathrm{d}}$ & 4.3 & 4336 & 1424 & n.d. & 264,000 & n.d. & n.d. & n.d. & n.d. \\
\hline Corta Atalaya acid pit lake, Spain ${ }^{\mathrm{e}}$ & 1.2 & 41,900 & 36,675 & n.d. & 158,730 & 18,689 & 1295 & $1,350,000$ & 5214 \\
\hline Rio Tinto River, Spain ${ }^{\mathrm{f}}$ & 1.4 & 15,000 & 4300 & n.d. & 22,000 & n.d. & n.d. & 210,000 & n.d. \\
\hline Koehler Breakdown, USA ${ }^{\mathrm{g}}$ & 2.8 & 2230 & 493 & n.d. & 8039 & n.d. & n.d. & n.d. & n.d. \\
\hline Virginia Canyon Mine, USA $^{\mathrm{g}}$ & 2.8 & 2940 & 381 & n.d. & 3687 & n.d. & n.d. & n.d. & n.d. \\
\hline Upper Rio Agrio, Patagonia, Argentina $^{\mathrm{h}}$ & 1.6 & 14,700 & 2650 & 1637 & 4780 & n.d. & n.d. & n.d. & n.d. \\
\hline Santa Lucia (mine effuents), sample L $2^{\mathrm{i}}$ & 2.7 & 6399 & 4330 & 370 & 191 & n.d. & n.d. & 95 & n.d. \\
\hline Santa Lucia (mine effuents), sample L $3^{\mathrm{i}}$ & 2.5 & 2149 & 264 & 860 & 5 & n.d. & n.d. & 1790 & n.d. \\
\hline Paradise Portal (PPREE1), CO, USA ${ }^{\mathrm{j}}$ & 5.3 & 1250 & 627 & 458 & 5 & 100 & $<45$ & 5 & 19 \\
\hline
\end{tabular}

n.d. not determined

${ }^{a}$ Average of six measurements

${ }^{\mathrm{b}}$ Migaszewski et al. (2015)

${ }^{\mathrm{c}}$ Nordstrom. Alpers (1999b)

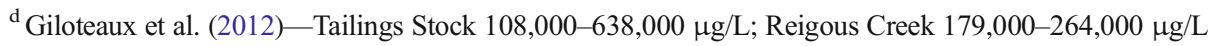

${ }^{\mathrm{e}}$ España et al. (2008)

${ }^{\mathrm{f}}$ Hudson-Edwards et al. (1999)

${ }^{\mathrm{g}}$ Bednar et al. (2005)

${ }^{\mathrm{h}}$ Gammons et al. (2005)

${ }^{\mathrm{i}}$ Romero et al. (2010)

${ }^{\mathrm{j}}$ Verplanck et al. (2001) 
Table 3 Average measured concentrations of REE, trace elements and sulfates vs. $\mathrm{pH}$ and alkalinity in the Silnica and Lubrzanka rivers adjacent to the Wiśniówka mining area

\begin{tabular}{|c|c|c|c|c|c|c|c|c|c|c|}
\hline Localization & $\mathrm{pH}$ & $\sum$ REE & $\mathrm{SO}_{4}{ }^{2}$ & Alkal. & $\mathrm{Fe}_{\text {total }}$ & As & Co & $\mathrm{Cr}$ & $\mathrm{Cu}$ & $\mathrm{Ni}$ \\
\hline & & $\mu \mathrm{g} / \mathrm{L}$ & $\mathrm{mg} / \mathrm{L}$ & $\mathrm{mg} / \mathrm{L}$ & $\mathrm{mg} / \mathrm{L}$ & $\mu \mathrm{g} / \mathrm{L}$ & $\mu \mathrm{g} / \mathrm{L}$ & $\mu \mathrm{g} / \mathrm{L}$ & $\mu \mathrm{g} / \mathrm{L}$ & $\mu \mathrm{g} / \mathrm{L}$ \\
\hline $\begin{array}{l}\text { Silnica } \text { R. }^{\mathrm{a}} \\
\quad(\text { no. } 10) \\
(N=3)\end{array}$ & 7.4 & 0.883 & 54 & 135 & 0.7 & 3.02 & 0.60 & 3.21 & 6.1 & 5.2 \\
\hline $\begin{array}{c}\text { Silnica } \mathrm{R}^{\mathrm{b}} \\
\quad(\text { no. } 11) \\
\quad(N=4)\end{array}$ & 7.2 & 1.160 & 61 & 167 & 2.8 & 4.86 & 12.48 & 3.06 & 13.18 & 20.0 \\
\hline $\begin{array}{l}\text { Silnica R. }{ }^{\mathrm{a}} \\
\quad \text { (no. 10) } \\
\quad \text { (March 14, } \\
\text { 2016) }\end{array}$ & 7.3 & 0.695 & 43 & 49 & 0.2 & 1.00 & 1.66 & 2.61 & 7.0 & 3.0 \\
\hline $\begin{array}{l}\text { Silnica R. } \\
\quad \text { (no. 11) } \\
\quad(\text { March 14, } \\
\text { 2016) }\end{array}$ & 6.9 & 5.625 & 134 & 13.9 & 0.4 & 2.25 & 30.9 & 1.79 & 86 & 31.8 \\
\hline $\begin{array}{l}\text { Lubrzanka } \text { R. }^{\mathrm{a}} \\
\quad(\text { no. } 8) \\
(N=2)\end{array}$ & 7.8 & 0.618 & 38 & 278 & 0.59 & 1.09 & 0.18 & 4.61 & 6.10 & 3.91 \\
\hline $\begin{array}{l}\text { Lubrzanka R. } \\
\quad(\text { no. } 9) \\
\quad(N=2)\end{array}$ & 7.8 & 0.657 & 40 & 256 & 0.83 & 1.04 & 2.12 & 3.69 & 17.10 & 8.36 \\
\hline $\begin{array}{l}\text { Lubrzanka R. } \\
\quad \text { (no. 8) } \\
\text { (March 14, } \\
\text { 2016) }\end{array}$ & 7.9 & 0.604 & 48 & 165 & $<0.2$ & 0.92 & 0.88 & 5.65 & 1.14 & 4.04 \\
\hline $\begin{array}{l}\text { Lubrzanka R. } \\
\text { (no. 9) } \\
(\text { March } 14, \\
\text { 2016) }\end{array}$ & 7.0 & 0.782 & 142 & 42 & $<0.2$ & 0.59 & 14.1 & 2.02 & 7.71 & 32.8 \\
\hline
\end{tabular}

For location of sampling sites see Fig. 1; concentrations of $\mathrm{PO}_{4}{ }^{3-}<0.1 \mathrm{mg} / \mathrm{L}$; Alkal. $-\mathrm{CaCO}_{3} \mathrm{mg} / \mathrm{L}$

$N$ number of samples

${ }^{\text {a }}$ Upstream (control water samples) from the Wiśniówka mining area

${ }^{\mathrm{b}}$ Downstream from the Wiśniówka mining area

${ }^{\mathrm{c}}$ Discharge of AMD waters into the Silnica River

${ }^{\mathrm{d}}$ Spills from tailings piles during heavy rains
Standards 53 Minerals 02753-AB, West Chester, PA 193810656 , USA), were used. This petrographic study was performed on raw and polished rock pieces (stereoscopic microscope), polished thin sections (polarizing microscope), and carbon-coated polished thin sections (SEM).

In addition, a few samples of pyrite were analyzed for trace metal(loid)s using the CETAC LSX 500+ laser ablation module coupled with the Perkin Elmer ICP-QMS ELAN DRC II instrument. The CETAC laser ablation module was equipped with the Q-switched Nd:YAG laser, frequency quadrupoled to $266 \mathrm{~nm}$. The single-line raster LA method was used. Sample surfaces were ablated with 250 shots at pulse energy of $>9 \mathrm{~mJ}$. The other optimized laser parameters included a pulse repetition rate of $10 \mathrm{~Hz}$, a delay between spots $2 \mathrm{~min}$, and a spot diameter of $25 \mu \mathrm{m}$. A time-resolved plot of signal for each element was integrated, and concentrations were computed using DigiLaz ${ }^{\mathrm{TM}}$-II operating software by CETAC. Operating conditions for the Perkin Elmer ICP-MS/MS ELAN DRC II instrument were as follows: low resolution, power $(\sim 1600 \mathrm{~W})$, and sample gas flow rate $(415 \pm 7 \mathrm{kPa}$ at
20 1/min Ar) adjusted to maximize atomic ion signal. The matrix-matched USGS microanalytical reference material, i.e., polymetal sulfide MASS-1, was used for quantitative analysis. The samples were analyzed for seven elements: As, $\mathrm{Cd}, \mathrm{Co}, \mathrm{Cu}, \mathrm{Ni}, \mathrm{Pb}$, and $\mathrm{Zn}$.

\section{Results and discussion}

\section{Geochemistry of waters}

The mean values of selected physicochemical parameters and the mean concentrations of sulfates, phosphates, REEs, and trace elements in the AMD and river waters from the Wiśniówka area are summarized in Tables 1,2 and 3. It should be underscored that due to the use of $0.45-\mu \mathrm{m}$ pore-sized filters, these contents reflect both true dissolved and partly colloidal element species.

The petrographic study has shown that the principal source of AMD waters is oxidation of crypto- to fine-grained pyrite 
that occurs in the form of thin veins cutting fine-grained quartzites and quartzitic sandstones or scattered assemblages within most clayey shale interbeds, and additionally in the Podwiśniówka quarry as cements of tectonic quartz-quartzite breccias (Fig. 3a-d). The pyrite grains lack other sulfide mineral inclusions. However, the SEM-EDS and LA-ICP-MS study of Podwiśniówka mineralized rock samples revealed the presence of micro-veins and alternating bands of arsenicrich pyrite (up to $8.2 \% \mathrm{As}$ ) within an arsenic-depleted pyrite matrix ( $<1 \%$ As) (Fig. 3e). The lack of As minerals and zonal distribution of arsenic may indicate that this element replaces both sulfur and iron in a pyrite crystal lattice, so not only sulfur as suggested by Kolker and Nordstrom (2001). Moreover, the pyrite comprises somewhat elevated concentrations of other elements (in parentheses maximum concentrations in $\mathrm{mg} / \mathrm{kg}$ ): $\mathrm{Cu}$ (1169), Ni (663), Zn (606), Co (121), Pb (107), and Cd (25) as indicated by the LA-ICP-MS study. The distinct determination coefficients $\left(R^{2}\right)$ for two pairs $\mathrm{Ni}-\mathrm{As}(0.95)$ and $\mathrm{Co}-$ As (0.68) may suggest that these two metals are linked to an As-rich pyrite variety as opposed to $\mathrm{Cu}$ which is uniformly distributed within the pyrite matrix. In contrast, $\mathrm{Ni}$ and $\mathrm{Co}$ substitute isomorphically for iron in a pyrite crystal lattice (Ribbe 1982). There is another significant constraint on the geochemistry of the acid waters examined. This is the lack of acid-consuming gangue and rock-forming minerals (except for subordinate muscovite displaying illitization) that have no attenuating effect on pyrite oxidation.

The oxidation of pyrite (Fig. 3b, f) has resulted in substantial lowering of $\mathrm{pH}$, release of minor and trace elements from its crystal lattice, and remobilization of metal(loid)s, especially REE, from associated clayey shale interbeds. These combined processes have the potential to form acid waters that contain high concentrations of $\mathrm{Al}, \mathrm{As}, \mathrm{Co}, \mathrm{Cr}, \mathrm{Cu}, \mathrm{Fe}$, and Ni. The most interesting is the Podwiśniówka acid pit lake that shows a very low $\mathrm{pH}(2.2-2.5)$ and very high concentrations of $\mathrm{SO}_{4}{ }^{2-}(2720-5460 \mathrm{mg} / \mathrm{L}), \mathrm{Fe}(545-1140 \mathrm{mg} / \mathrm{L}), \mathrm{Al}$ (86.2 mg/L), As (9603-24,883 $\mu \mathrm{g} / \mathrm{L})$, Co (1317-3458 $\mu \mathrm{g} / \mathrm{L})$, $\mathrm{Cr}(753-2047 \mu \mathrm{g} / \mathrm{L}), \mathrm{Cu}(6307-18,879 \mu \mathrm{g} / \mathrm{L}), \mathrm{Ni}(1168-$ $3127 \mu \mathrm{g} / \mathrm{L})$, and REE $(589-1341 \mu \mathrm{g} / \mathrm{L})$. These contents exceed the permissible levels for industrial wastewater standards set by the Regulation of the Minister of Environmental Protection (2014) (see Table 1). Of the elements mentioned above, $\mathrm{As}, \mathrm{Fe}_{\text {total }}, \mathrm{Cu}, \mathrm{Al}, \mathrm{Ni}, \mathrm{Cr}$, and $\mathrm{Co}$ concentrations exceed these limits by a factor of 96 to 249 (As), 54 to 114 (Fe), 13 to $38(\mathrm{Cu}), 29(\mathrm{Al}), 2$ to $6(\mathrm{Ni}), 1.5$ to $4(\mathrm{Cr})$, and 1.3 to 3.5 (Co). By comparison, $\mathrm{SO}_{4}{ }^{2-}$ contents were 5 to 11 times
Fig. 3 Stereoscopic micrographs of a tectonic quartzite-quartz tectonic breccia cemented with pyrite and $\mathbf{b}$ same tectonic breccia with needle-shaped secondary iron sulfates. Petrographic micrographs of $\mathbf{c}$ pyrite-quartz veinlet cutting quartzite, reflected light, 1 nicol, and $\mathbf{d}$ tectonic quartzitequartz breccia cemented with crypto- and micro-grained pyrite, reflected light, 1 nicol. SEM images of e light gray As-rich pyrite forming veinlets and alternating bands with darker As-depleted pyrite (taken by L. Giro) and $\mathbf{f}$ weathered pyrite grain embedded in quartz veinlet (taken by L. Giro). $P y$ pyrite, $A s-P y$ arsenicrich pyrite, $\mathrm{Fe}$-sulf iron sulfates, Qtz quartz, Qtzt quartzite

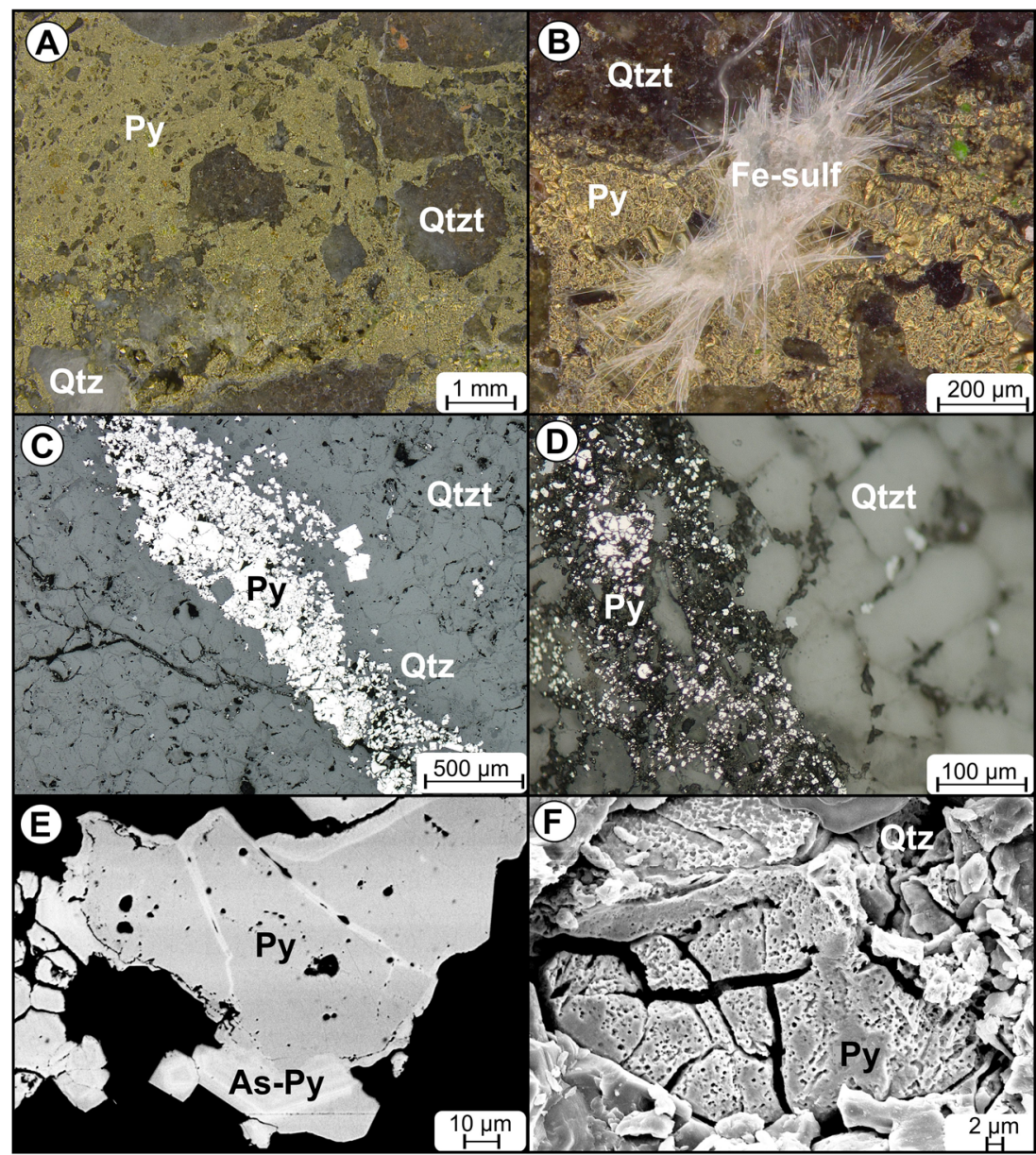


higher than the set level of $500 \mathrm{mg} / \mathrm{L}$. However, the high concentrations of As in the Podwiśniówka acid pit lake varying from 9603 to $24,883 \mu \mathrm{g} / \mathrm{L}$, with a mean of $15,416 \pm 5197 \mu \mathrm{g} / \mathrm{L}$ are of greater environmental concern (Tables 1 and 2). Along with strong acid pools, in which As contents reach $369,726 \mu \mathrm{g} / \mathrm{L}$, these waters are among the most distinctive As-rich AMD surface waters throughout the world (Table 2).

In the most representative Podwiśniówka acid pit lake, which is presently not influenced by mining and processing operations, the lowest concentrations of $\mathrm{SO}_{4}{ }^{2-}, \mathrm{Co}, \mathrm{Cr}, \mathrm{Cu}$, $\mathrm{Ni}$, and the lowest values of TDS, salinity, and EC were noted in the winter of 2014 whereas the highest concentrations of $\mathrm{Fe}$ and $\mathrm{Mn}$ were observed in the summer of 2014. The highest levels of As and Ni were in turn found in the fall of 2014 and 2015. However, more data are needed for further discussion.

The three biggest acid water bodies (Table 1) showed diverse chemistry which is closely linked to mineralogy and petrology of exposed rock formations and different pyrite generations (study in progress). The lowest $\mathrm{pH}$ and the high metal(loid) concentrations in the Podwiśniówka acid pit lake water are due primarily to a greater amount of pyrite and presumably hematite and goethite. The REE-bearing minerals (gorceixite, florencite, xenotime, zircon) occur in clayey shale and tuffite interbeds of the Podwiśniówka quarry as shown by the previous studies (Migaszewski et al. 2007b; Migaszewski and Gałuszka 2010). For instance, some black clayey shale interbeds contain up to $454,66 \mathrm{mg} / \mathrm{kg}$ REE as opposed to pyrite which is substantially depleted in REE (22.02 to $90,65 \mathrm{mg} / \mathrm{kg}$ ) (archival data). This is also reflected by high concentrations of REE (mean of $993 \pm 282 \mu \mathrm{g} / \mathrm{L}$ ) in the Podwiśniówka acid pit lake (Table 1). It is noteworthy that these concentrations are among the highest found in AMD streams, lakes, and ponds across the world (Table 2). These include acid mine effluents of the abandoned $\mathrm{Zn}-\mathrm{Pb}$ mine of Santa Lucia in western Cuba (370-860 $\mu \mathrm{g} / \mathrm{L}$ ) (Romero et al. 2010 ) or stream waters of a $\mathrm{Cu}-\mathrm{Pb}-\mathrm{Zn}$ mining area in Metalliferous Hills, Italy $(929 \mu \mathrm{g} / \mathrm{L}$ ) (Protano and Riccobono 2002). It is noteworthy that the highest concentration of REE $(29 \mathrm{mg} / \mathrm{L})$ at a $\mathrm{pH}$ of 3.6 was reported in groundwater from the Osamu Utsumi uranium mine, Brazil (Miekeley et al. 1992).

The distinctly lower contents of As and trace metals were recorded in the Wiśniowka Duża acid pit sump (Table 1). The only exception was copper whose concentrations (878$2508 \mathrm{mg} / \mathrm{L}$ ) exceeded the permissible limit by a factor of $2-$ 5. In contrast, the $\mathrm{SO}_{4}{ }^{2-}$ concentrations $(423$ to $882 \mathrm{mg} / \mathrm{L}$, mean of $575 \pm 184 \mathrm{mg} / \mathrm{L}$ ) varied around the permissible value of $500 \mathrm{mg} / \mathrm{L}$ (Regulation of the Minister of Environmental Protection 2014). Except for sulfate (494 to $648 \mathrm{mg} / \mathrm{L}$, mean of $534 \pm 64 \mathrm{mg} / \mathrm{L}$ ), the Wiśniówka Mała acid pit lake water showed in turn the lowest concentrations of trace metal(loid)s (Table 1). Nonetheless, the levels of $\mathrm{SO}_{4}{ }^{2-}$ were higher than those recorded during 2005-2006 (108-192 $\mathrm{mg} / \mathrm{L})$ prior to washing of pyrite-rich quartzite aggregates from the Podwiśniówka quarry (Migaszewski et al. 2009).

It is interesting to note that the similar trace element concentrations to Wiśniówka Duża and Wiśniówka Mała acid pit waters were exhibited by historic tailings acid ponds (Table 1). Also, in this case, the $\mathrm{SO}_{4}{ }^{2-}$ concentrations were in the range of 256 to $976 \mathrm{mg} / \mathrm{L}$ due to heterogeneity of stockpiled mine waste material. The easternmost acid pond showed the lowest $\mathrm{pH}$ and the highest concentrations of sulfates and metal(loid)s, particularly $\mathrm{Cu}$ reaching $839 \mu \mathrm{g} / \mathrm{L}$. This suggests that this portion of the tailings pile consists of Wiśniówka Duża waste material.

These three major acid water bodies of Podwiśniówka, Wiśniówka Duża, and Wiśniówka Mała have revealed some variations in sulfate and metal(loid) concentrations. The only exception was the $\mathrm{pH}$ that showed relatively stable values. Aside from $\mathrm{Pb}, \mathrm{Mn}, \mathrm{As}$, and $\mathrm{Zn}$ in the Podwiśniówka acid pit lake and Wiśniówka Duża acid pit sump, the concentrations of remaining elements and $\mathrm{SO}_{4}{ }^{2-}$ did not show extreme variations (below a factor of 3). These may be induced by different influxes of pyrite oxidation products, changes in temperature, insolation, and precipitation patterns (rainfalls, droughts, ice cover), bacterial activity, secondary mineral transformations, sorption/desorption of trace elements, etc. (Acero et al. 2006; Leybourne and Johannesson 2008).

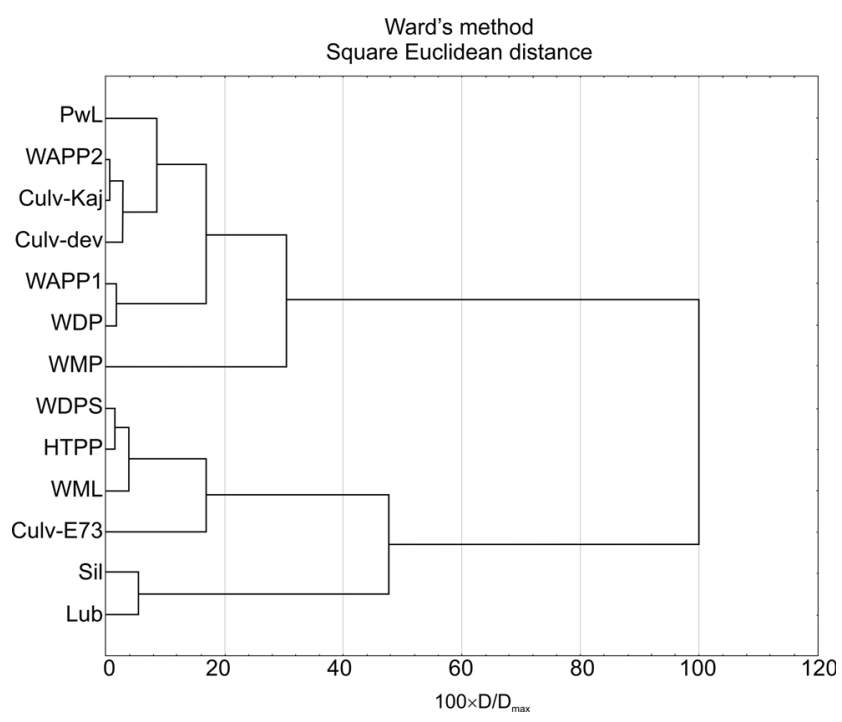

Fig. 4 Spatial (site) dendrograms of AMD waters of the Wiśniówka mining area vs. Silnica and Lubrzanka rivers. $P w L$ Podwiśniówka pit lake (no. 1 in Fig. 1), WAPP2 Wiśniówka aggregate processing plant pool 2 (no. 7), Culv-Kaj culvert under railroad track in Kajetanów (no. 14), Culv-dev culvert under housing development (no. 13), WAPPI Wiśniówka aggregate processing plant pool 1 (no. 6), WDP Wiśniówka Duża pool (no. 5), WMP Wiśniówka Mała pond (not shown in Fig. 1, between nos. 3 and 13), WDPS Wiśniówka Duża pit sump pond (no. 2), HTPP historic tailings pile ponds (no. 4), WML Wiśniówka Mała pit lake (no. 3), Culv-E73 culvert under highway E-73 (no. 12), Sil Silnica River downstream (no. 11), Lub Lubrzanka River downstream (no. 9). For location of sampling points, see Fig. 1 
The extremely metal(loid)-rich acid pools (sampling point nos. 5-7 in Fig. 1) displayed the $\mathrm{pH}$ in the range of 1.7 to 2.8 and very high concentrations of $\mathrm{SO}_{4}{ }^{2-}, \mathrm{Al}, \mathrm{As}, \mathrm{Co}, \mathrm{Cr}, \mathrm{Cu}, \mathrm{Fe}$, $\mathrm{Mn}, \mathrm{Ni}$, and $\mathrm{Zn}$ (Table 2). Most of these parameters exceeded the set limits by a factor of several tens to hundreds, for instance concentrations of arsenic varied from 16,721 to $369,726 \mu \mathrm{g} / \mathrm{L}$. It is interesting to note that REE concentrations were also very high reaching $6288 \mu \mathrm{g} / \mathrm{L}$. However, these extreme values were lower than those (up to $8.15 \mathrm{mg} / \mathrm{L} \mathrm{REE}$ ) found in underground waters impacted by AMD in an abandoned uranium mining area of Eastern Thuringia, Germany (Merten et al. 2007).

There are no statistically significant correlations between the $\mathrm{pH}$ and element concentrations in the Wiśniówka AMD lakes, ponds, and pit sump. The best example is arsenic that does not exhibit any correlation with $\mathrm{pH}$ or $\mathrm{SO}_{4}{ }^{2-}$ concentrations. The other studies have also indicated relative mobility of this metalloid over a wide range of $\mathrm{pH}$ and redox conditions (e.g., Smedley and Kinniburgh 2002; Courtin-Nomade et al. 2005). However, the $\mathrm{pH}$ shows correlation with EC $\left(R^{2}=-0.86\right)$ and in turn EC with As (0.97) and Ni (0.88) whereas As with Ni (0.86). The highest determination coefficients $\left(R^{2}>0.90\right)$ are noted for the following pairs: $\mathrm{SO}_{4}{ }^{2-}-$ $\mathrm{Fe}_{\text {total }}(0.97), \mathrm{Mn}-\mathrm{Pb}$ (0.97), Co-Sc (0.96), Al-Cd (0.95), Co$\mathrm{Ni}(0.94), \mathrm{Co}-\mathrm{Cu}(0.93)$, and $\mathrm{Ni}-\mathrm{Sc}(0.93)$. The strong correlations between $\mathrm{As}$ or $\mathrm{Ni}$ and $\mathrm{EC}$ may also point to a relationship of these two elements with dissolved and some colloid fractions $(<0.45 \mu \mathrm{m})$. In contrast to As, Ni should not have a high affinity to amorphous hydrous ferric oxides under acidic conditions. This metal may show affinity with some clay minerals (illite, kaolinite, and mixed-layered illite-smectite) that were found in the sediment of the former Podwiśniówka acid pit pond (Migaszewski et al. 2008), but this issue needs further studying.
In contrast to the acid water bodies described above, the Lubrzanka and Silnica rivers upstream and downstream from the Wiśniówka mining area revealed the $\mathrm{pH}$ values in the range of 6.9 to 7.9 and simultaneously low concentrations of $\mathrm{SO}_{4}{ }^{2-}$ and trace amounts of metal(loid)s, including As (0.59$4.86 \mu \mathrm{g} / \mathrm{L})$ and REE $(0.604-5.625 \mu \mathrm{g} / \mathrm{L})$. An insignificant drop in the $\mathrm{pH}$ and alkalinity, and a raise in concentrations of some metal(loid)s were noted during rainy season in March of 2016. This was caused by a small inflow of AMD waters as a result of long-lasting rainfall.

The element relationship between different acid pit water bodies and the Silnica or Lubrzanka rivers may also be evidenced by a spatial (site) variable dendrogram that groups these objects into two clusters at the linkage distance (Dlink/ Dmax $\times 100)<51$ (Fig. 4): (i) Podwiśniówka lake (PwL), metal(loid)-rich acid pools (WAPP1, WAPP2, WDP), culverts (Culv-Kaj, Culv dev) (<15) and metal(loid)-rich acid pool (WMP) (<30), and (ii) Wiśniówka Duża pit sump (WDPS), Wiśniówka Mała lake (WML), and historic tailings pile ponds (HTPP) $(<5)$ and culvert $(<15)$. The acid pools of the first cluster form seeps at the foot of Podwiśniówka aggregate and waste material piles, hence their close relationship with geochemistry of the Podwiśniówka acid pit lake water. These seeps also flow down through two culverts (Culv-Kaj, Culv dev). The remaining AMD water bodies are in turn grouped together in the second cluster giving evidence for interrelated Wiśniówka Duża and Wiśniówka Mała mining and processing operations. Nonetheless, none of these two groups do exhibit any linkage to Silnica (Sil) and Lubrzanka (Lub).

\section{NASC-normalized REE patterns}

The average measured LREE/HREE and NASC-normalized REE concentration ratios $\left(\mathrm{HREE}_{\mathrm{NASC}} \mathrm{LREE}_{\mathrm{NASC}}, \mathrm{La}_{\mathrm{NASC}}\right.$

Table 4 Average measured and NASC-normalized REE concentration ratios in AMD water bodies of the Wiśniówka mining area and adjacent rivers

\begin{tabular}{|c|c|c|c|c|c|c|c|}
\hline Location & $\begin{array}{l}\text { Pw pit lake } \\
(N=6)\end{array}$ & $\begin{array}{l}\text { WD pit sump } \\
(N=5)\end{array}$ & $\begin{array}{l}\text { WM pit lake } \\
(N=5)\end{array}$ & $\begin{array}{l}\text { Historic tailings pit } \\
\text { ponds }(N=6)\end{array}$ & $\begin{array}{l}\text { Silnica } \mathrm{R} . \\
\text { downstream } \\
(N=1)\end{array}$ & $\begin{array}{l}\text { Lubrzanka R. } \\
\text { downstream }(N=1)\end{array}$ & $\begin{array}{l}\text { Clayey shales Pw } \\
\text { quarry }(N=9)\end{array}$ \\
\hline $\begin{array}{l}\mathrm{REE}_{(\mathrm{La}-\mathrm{Lu})} \\
\quad(\mu \mathrm{g} / \mathrm{L})\end{array}$ & 829 & 174 & 95 & 214 & 5.62 & 0.80 & $348.54^{\mathrm{a}}$ \\
\hline $\begin{array}{l}\text { LREE }_{\text {measured }} \\
\text { HREE }_{\text {measured }}\end{array}$ & 2.58 & 1.49 & 2.21 & 1.91 & 2.12 & 10.85 & $15.26^{\mathrm{a}}$ \\
\hline $\begin{array}{l}\text { HREE }_{\text {NASC }^{\prime}} \\
\text { LREE }_{\text {NASC }}\end{array}$ & 1.76 & 3.52 & 2.34 & 2.74 & 3.57 & 0.86 & 0.50 \\
\hline $\mathrm{La}_{\text {NASC }} / \mathrm{Yb}_{\text {NASC }}$ & 0.34 & 0.12 & 0.32 & 0.17 & 0.52 & 0.79 & 2.76 \\
\hline $\begin{array}{l}\mathrm{Sm}_{\mathrm{NASC}} \\
\mathrm{Yb}_{\mathrm{NASC}}\end{array}$ & 1.97 & 0.71 & 1.25 & 1.00 & 0.41 & 0.47 & 2.56 \\
\hline $\mathrm{Ce} / \mathrm{Ce}_{\mathrm{NASC}}{ }^{a}$ & 0.99 & 1.19 & 0.96 & 0.96 & 0.42 & 0.96 & 1.15 \\
\hline $\mathrm{Eu} / \mathrm{Eu}_{\mathrm{NASC}}{ }^{\mathrm{a}}$ & 1.38 & 0.92 & 1.17 & 1.07 & 0.65 & 1.27 & 1.74 \\
\hline $\mathrm{Gd} / \mathrm{Gd}_{\text {NASC }}{ }^{\mathrm{a}}$ & 1.46 & 1.34 & 1.45 & 1.37 & 1.53 & 0.92 & 1.15 \\
\hline $\mathrm{Tb} / \mathrm{Tb}_{\mathrm{NASC}}{ }^{\mathrm{a}}$ & 1.30 & 1.40 & 1.41 & 1.42 & 1.39 & 1.12 & 0.82 \\
\hline
\end{tabular}

${ }^{a}$ Excluding Lu concentration 

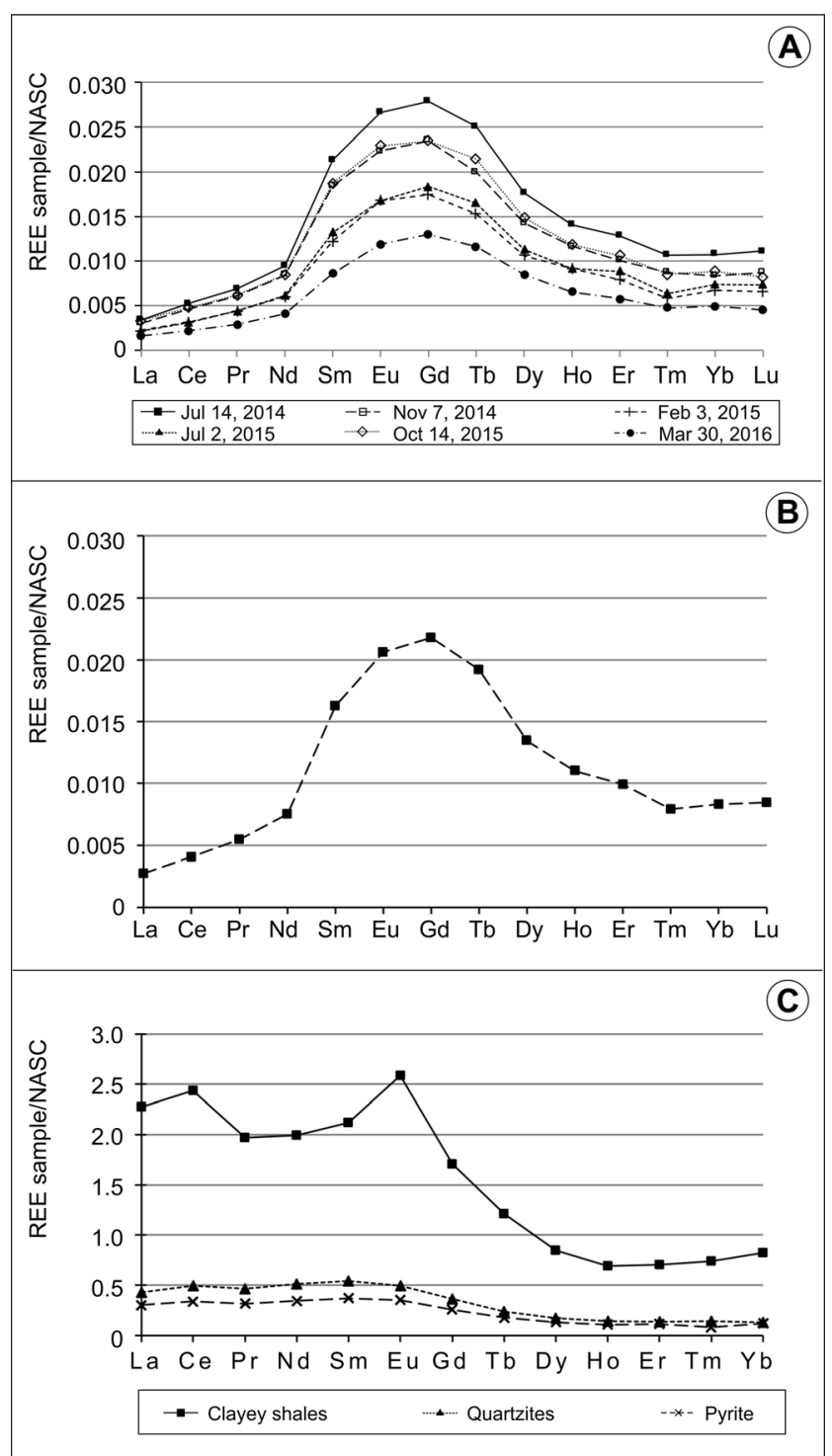

Fig. 5 NASC-normalized REE concentration patterns of Podwiśniówka new acid pit lake water: $\mathbf{a}$ a series of six measurements and $\mathbf{b}$ mean value of six measurements, $\mathbf{c}$ patterns of rocks and pyrite (Migaszewski et al. 2014)

$\mathrm{Yb}_{\text {NASC }}, \mathrm{Sm}_{\mathrm{NASC}} / \mathrm{Yb}_{\text {NASC }}$ ) are summarized in Table 4. In all these water samples, the measured LREE prevail over HREE with ratios in the range of 1.49 (Wiśniówka Duża pit sump) to 2.58 (Podwiśniówka acid pit lake). Nonetheless, the reverse relationship is observed when comparing their shalenormalized equivalents: the $\mathrm{HREE}_{\mathrm{NASC}} / \mathrm{LREE}_{\mathrm{NASC}}$ ratio varies from 1.76 (Podwiśniówka pit lake) to 3.52 (Wiśniówka Duża pit sump). The same relationship is observed between shale-normalized $\mathrm{La} / \mathrm{Yb}$ and $\mathrm{Sm} / \mathrm{Yb}$ ratios, for instance the $\mathrm{La}_{\mathrm{NASC}} / \mathrm{Yb}_{\mathrm{NASC}}$ ratio is in the range of 0.12 (Wiśniówka Duża) to 0.34 (Podwiśniówka) (Table 4). The lowest $\mathrm{Sm}_{\mathrm{NASC}} / \mathrm{Yb}_{\text {NASC }}$ ratio (0.71) in the Wiśniówka Duża pit sump suggests that LREE are scavenged mostly by $\mathrm{Fe}$ - and Mn-oxide/oxyhydroxide and clay colloids (Sholkovitz 1995;
Johannesson and Zhou 1999). However, experiments done on kaolinite and Na-montmorillinite have shown that the REE sorption is more complex and is controlled by the cation exchange capacity of clay minerals, $\mathrm{pH}$, and ionic strength, for instance at higher ionic strength $(0.5$ vs. $0.025 \mathrm{M})$, the HREE were more sorbed than the LREE (Coppin et al. 2002). This means that the fractionation of REE between solid and solution phases still remains an unresolved issue.

The predominance of HREE over LREE is also evidenced by the normalized to NASC mean REE concentration patterns of Wiśniówka acid lakes and ponds (Figs. 5 and 6). This may be explained by preferential withdrawing of LREE from solution as a result of scavenging/adsorption by $\mathrm{Fe}$ - and Mn-oxide/ oxyhydroxide colloids, poorly soluble secondary minerals (including jarosite), or organic matter-coated suspended detrital mineral particles (e.g., Gammons et al. 2003; Romero et al. 2010). Experiments document that despite considerable Fe(II) oxidation, REE remain in solution at a $\mathrm{pH}<5.1$, but in the range of 5.1-6.6, the HREE are removed from the water column compared to LREE and are partitioned into the ferric iron solid phases (Verplanck et al. 2004).

The Podwiśniówka acid pit lake water reveals a characteristic roof-shaped NASC-normalized REE concentration pattern with the prevailing MREE, especially $\mathrm{Gd}, \mathrm{Eu}$, and $\mathrm{Tb}$ (Fig. 5a, b). The predominance of these three elements is enhanced by the $\mathrm{Gd} / \mathrm{Gd}_{\mathrm{NASC}}, \mathrm{Eu} / \mathrm{Eu}_{\mathrm{NASC}}$, and $\mathrm{Tb} / \mathrm{Tb}_{\mathrm{NASC}}$ ratios: $1.46,1.38$, and 1.30 , respectively (Table 4 ). Moreover, this convex-up REE profile suggests that MREE are either more easily leached from the host rocks or are more dissolved under the $\mathrm{pH}$ and redox conditions that prevail in AMD ponds and lakes (Leybourne et al. 2000). The MREE enrichment in AMD waters has been reported worldwide by many authors (e.g., Johannesson and Zhou 1999; Gimeno Serrano et al. 2000; Worrall and Pearson 2001; Zhao et al. 2007; Pérez-López et al. 2010; Grawunder et al. 2014). However, the results derived from these studies suggest that other explanations of this phenomenon must be also considered, for example dissolution of primary minerals uraninite (McLennan 1994), apatite (Hannigan and Sholkovitz 2001), and pyrite (e.g., Grawunder et al. 2014) — and/or secondary minerals - Fe-oxyhydroxysulfates (Pérez-López et al. 2010) or Fe-Mn-oxides and oxyhydroxides (Johannesson and Zhou 1999).

The clayey shales show a distinct predominance of NASCnormalized LREE over HREE mean concentrations $(N=9)$ with a distinct positive Eu anomaly (Fig. $5 \mathrm{c}$ ). The $\mathrm{Eu} / \mathrm{Eu}^{*}{ }_{\text {NASC }}$ ratio amounts to 1.74 . This is also enhanced by the HREE $_{\text {NASC }}$ LREE $_{\text {NASC }}$ ratio averaging 0.50 . It is interesting to note that Podwiśniówka clayey shales and acid pit lake water do not reveal any $\mathrm{Ce}$ anomaly which is also evidenced by $\mathrm{Ce} /$ $\mathrm{Ce}^{*}{ }_{\text {NASC }}$ ratios averaging 1.15 and 0.99 , respectively. This 


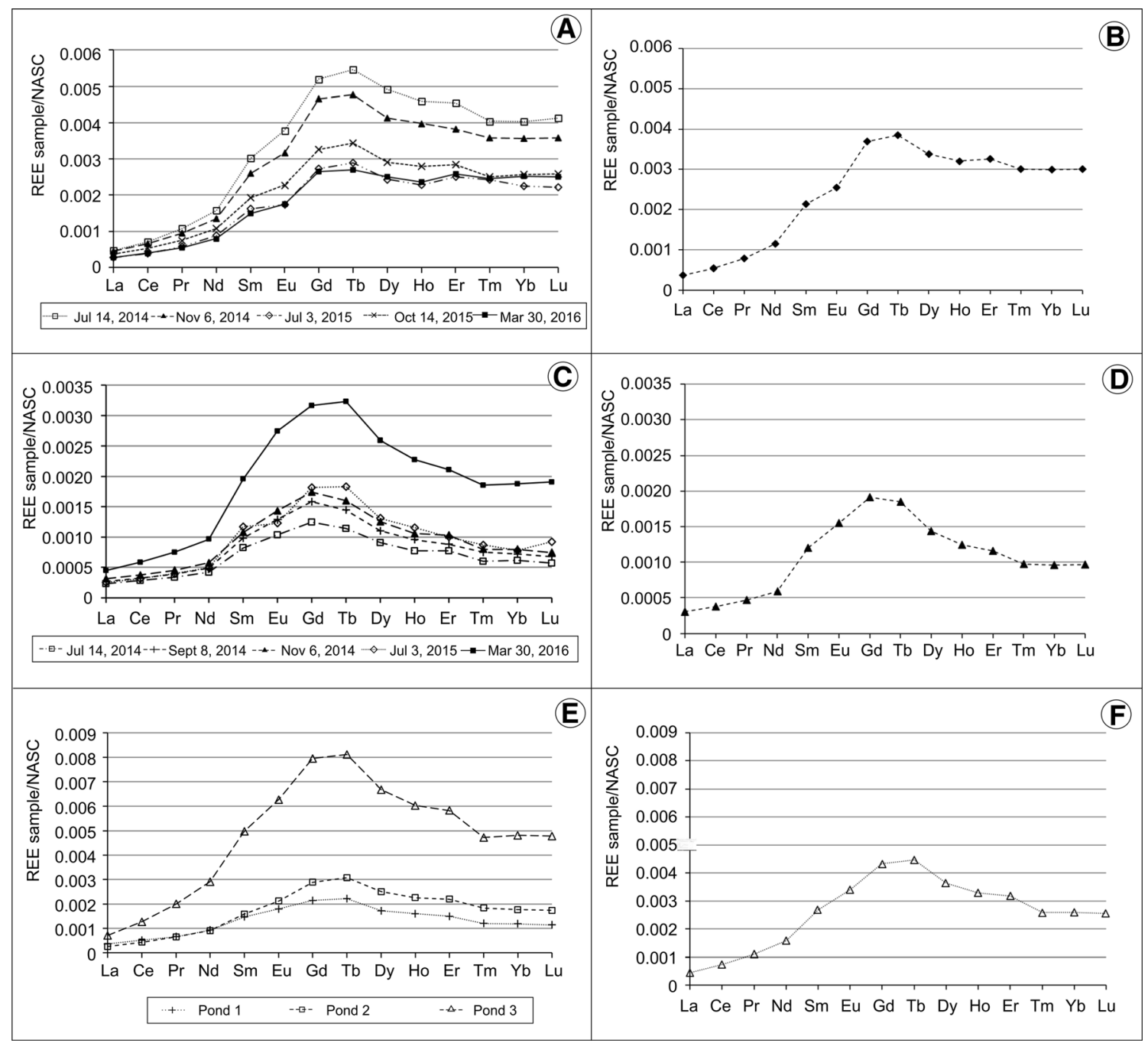

Fig. 6 NASC-normalized REE concentration patterns of different acid water bodies: Wiśniówka Duża pit sump water: a a series of five measurements, b mean value of five measurements; Wiśniówka Mała

acid pit lake (western part) water: $\mathbf{c}$ a series of five measurements, $\mathbf{d}$ mean value of five measurements; acid pond waters on historic tailings piles: e three selected ponds, $\mathbf{f}$ mean value of three measurements

may suggest an invariable transfer of the REE patterns from these rocks to the pit lake water with insignificant modification by diverse solubility of REE-bearing minerals and different geochemical processes (sorption/desorption, dissolution).

The determination coefficients $\left(R^{2}\right)$ between concentrations of individual REE and $\mathrm{Mn}, \mathrm{Fe}, \mathrm{Al}$, and $\mathrm{SO}_{4}{ }^{2-}$ in the Wiśniówka AMD waters are presented in Table 5. It is interesting to note that Pr through Dy show very strong correlations ( $\geq 0.85)$ with Mn. Of the LREE group, Ce and La reveal distinctly stronger correlation with $\mathrm{Al}\left(R^{2}=0.85\right.$ and 0.86$)$ than with $\mathrm{Fe}\left(R^{2}=0.38\right.$ and 0.35$)$. The factor analysis for all the measured parameters does not exhibit isolated structures within the dataset. $\mathrm{Al}, \mathrm{La}$, and Ce have been related to factor 2 . These results contradict those obtained by Gammons et al. (2005) who reported selective partitioning of HREE to Al oxyhydroxides. In contrast, the HREE and Sm have far greater affinities to $\mathrm{Fe}\left(R^{2}=\sim 0.75\right)$ than to $\mathrm{Al}\left(R^{2}=\sim 0.48\right)$. On the other hand, different studies have also shown that the REE may occur in the water as prevailing free metal cations (Leybourne et al. 2000) and sulfate complexes $\mathrm{LnSO}_{4}{ }^{+}$ (Johannesson and Lyons 1995). The last option must be considered for the Wiśniówka AMD waters due to the strong correlation of HREE with $\mathrm{SO}_{4}{ }^{2-}$ ions; $R^{2}$ varies from 0.70 (Gd) to $0.87(\mathrm{Lu})$ (Table 5). This REE fractionation may be coupled at least partly with multiple adsorption-desorption reactions and/or ion exchange between the water column and precipitates, colloids, or secondary minerals as reported by other authors (e.g., Johannesson and Lyons 1995; Gimeno Serrano et al. 2000; Romero et al. 2010).

In contrast, the Wiśniówka Duża acid pit sump exhibits the totally different step-shaped NASC-normalized REE concentration pattern with the most distinct predominance of HREE and positive $\mathrm{Tb}$ and $\mathrm{Gd}$ positive anomalies (Fig. 6a, b). This is confirmed by the $\mathrm{Tb} / \mathrm{Tb}^{*}{ }_{\text {NASC }}$ and 
$\mathrm{Gd} / \mathrm{Gd}^{*}{ }^{*} \mathrm{NASC}$ ratios, which are 1.40 and 1.34 , respectively (Table 4). This also suggests that the younger Upper Cambrian beds (compared to the Podwiśniówka and Wiśniówka Mała quarries) must reveal different mineralogy and geochemistry, which is also evidenced by the lack of excessive amounts of $\mathrm{As}, \mathrm{Co}, \mathrm{Cr}, \mathrm{Fe}$, and $\mathrm{Ni}$ (see the "Geochemistry of waters section).

Like the Podwiśniówka acid pit lake, the Wiśniówka Mała acid pit lake shows nearly the same shale-normalized mean REE concentration pattern with the predominance of $\mathrm{Gd}$ and $\mathrm{Tb}$ (Fig. 6c, d). Both elements exhibit distinct $\mathrm{Gd} / \mathrm{Gd}^{*}{ }_{\text {NASC }}$ (1.45) and $\mathrm{Tb} / \mathrm{Tb}^{*}{ }_{\text {NASC }}(1.41)$ ratios. This overlaps the NASCnormalized REE profile of the former Podwiśniówka acid pit pond that was located at a higher mining bench compared to the current Podwiśniówka acid pit lake (Migaszewski et al. 2014). It is noteworthy that this pattern has changed since July 3 of 2015 due to the use of Wiśniówka Mała pit lake water for washing of crushed aggregate and dumping waste material from the Wiśniówka Duża (Fig. 6c, d). It is interesting to note that this change has not been accompanied by a distinct shift in the Wiśniówka Mała water chemistry.

The $\mathrm{Ce} / \mathrm{Ce}_{\mathrm{NASC}}{ }^{*}$ ratios in the acid reservoir waters varied from 0.96 (Wiśniówka Mała) to 1.19 (Wiśniówka Duża). If there is any anomaly, then it is rather a small positive one with 1.19. Except for Podwiśniówka, the Eu/Eu ${ }^{*}{ }_{\text {NASC }}$ ratios varied from 0.92 (Wiśniówka Duża) to 1.17 (Wiśniówka Mała).

The historic tailings acid ponds and intermittent strong acid pools display the NASC-normalized REE patterns bodies (Fig. 6e, f) similar to those of Podwiśniówka and/or Wiśniówka Duża water. Moreover, they generally exhibit intermediate NASC-normalized REE concentration ratios with the predominance of $\mathrm{Tb} / \mathrm{Tb}^{*}{ }_{\text {NASC }}(1.42)$ and $\mathrm{Gd} / \mathrm{Gd}^{*}{ }_{\text {NASC }}$

\begin{tabular}{llllll}
\cline { 2 - 5 } $\begin{array}{l}\text { Table 5 Determination } \\
\text { coefficients }\left(R^{2}\right) \text { between } \\
\text { concentrations of }\end{array}$ & $\mathrm{REE}$ & $\mathrm{Mn}$ & $\mathrm{Fe}_{\text {total }}$ & $\mathrm{Al}$ & $\mathrm{SO}_{4}{ }^{2-}$ \\
\cline { 2 - 6 } individual REE and Mn, & $\mathrm{La}$ & 0.76 & 0.35 & 0.86 & 0.39 \\
Fe, Al, $\mathrm{SO}_{4}{ }^{2-}$ in the & $\mathrm{Ce}$ & 0.77 & 0.36 & 0.85 & 0.42 \\
Wiśniówka AMD water & $\mathrm{Pr}$ & 0.85 & 0.52 & 0.73 & 0.53 \\
bodies & $\mathrm{Nd}$ & 0.88 & 0.62 & 0.62 & 0.61 \\
& $\mathrm{Sm}$ & 0.89 & 0.74 & 0.36 & 0.70 \\
& $\mathrm{Eu}$ & 0.90 & 0.72 & 0.37 & 0.67 \\
& $\mathrm{Gd}$ & 0.89 & 0.73 & 0.41 & 0.70 \\
& $\mathrm{~Tb}$ & 0.88 & 0.74 & 0.47 & 0.73 \\
& $\mathrm{Dy}$ & 0.85 & 0.73 & 0.50 & 0.75 \\
& $\mathrm{Ho}$ & 0.82 & 0.74 & 0.52 & 0.77 \\
& $\mathrm{Er}$ & 0.79 & 0.74 & 0.52 & 0.79 \\
& $\mathrm{Tm}$ & 0.75 & 0.74 & 0.52 & 0.80 \\
& $\mathrm{Yb}$ & 0.72 & 0.77 & 0.46 & 0.83 \\
& $\mathrm{Lu}$ & 0.68 & 0.80 & 0.41 & 0.87 \\
\hline
\end{tabular}

Values showing the strongest $R^{2}(\geq 0.85)$ are italicized
(1.37) (Table 4). Some variations in the NASC-normalized REE patterns and ratios depend on the localization of ponds on historic tailings which are built of mine and processing wastes derived from different sources. This issue is discussed in more detail in the "Element fingerprints of mining operations" section.

\section{Element fingerprints of mining operations}

The study area is a unique AMD site where only rocks (quartzites) are quarried whereas pyrite constitutes only subordinate mineralization of no economic value. This is in contrast to most AMD sites throughout the world which are closely linked to metal ore or coal deposits (e.g., Monterroso et al. 1998; Plumlee et al. 1999; Hudson-Edwards et al. 1999; Nordstrom and Alpers 1999a, b; Protano and Riccobono 2002; Gammons et al. 2003; Zhao et al. 2007; Romero et al. 2010; Migaszewski et al. 2015). Another distinguished feature of the Wiśniówka mining area is a lack of other sulfide minerals and effectively buffering gangue minerals and rocks. As discussed in the previous two sections, the three acid pit water bodies exhibit different chemistry that generally correlates with the geologic makeup of the quarries. This also finds its reflection in the specific roof- and step-shaped (Podwiśniówka vs. Wiśniówka Duża) NASC-normalized REE concentration patterns and characteristic enrichment of Podwiśniówka rock series (and waters) in $\mathrm{As}, \mathrm{Co}, \mathrm{Cr}, \mathrm{Cu}$, and $\mathrm{Ni}$ that enable identification of potentially hazardous places.

The Podwiśniówka tailings and crushed aggregate stockpiled at a depository site near the Wiśniówka Mała quarry and near the Wiśniówka aggregate processing plant give rise for concern. These piles are a source of seeps forming strong acid and As- and metal-rich pools. These in turn are washed away by rainfalls outside of the mining area occasionally jeopardizing the neighboring river systems. Similar potential threats posed by seeps to the environment have been studied in other AMD sites using mining-related metal(loid)s (e.g., Mighanetara et al. 2009). The REE patterns were also employed to identify seepage water from historic tailings piles as a source of contamination of valley sediments at Ronneburg mining area (e.g., Merten et al. 2005).

The comparison of geochemical signatures of historic tailings ponds with those of the three acid pit water bodies has indicated that these tailings piles are built of mine wastes derived primarily from extraction of Wiśniówka Mała and Wiśniówka Duża quartzites. In addition, the chemistry of some pools that form in the Podwiśniówka and Wiśniówka Duża quarries provides information on unexposed pyrite occurrences that may locally occur within the intensively folded and faulted Wiśniówka massif. This may give a clue to the future mining operations in a tectonically affected area. 


\section{Interactions of mine drainage with the environment}

The results of this study show an insignificant impact of untreated AMD waters from the Wiśniówka Duża pit sump and Wiśniówka Mała lake on the neighboring river systems due to the presence of carbonate rocks (limestones, marly shales, limestone conglomerates) in the Silnica River bed and partly in an upper course of the Lubrzanka River. This is the reason why the waters of these streams have an effective buffering capacity (Table 3). For example, close to the confluence of a ditch (draining the Wiśniówka Duża pit sump; sampling point nos. 5 and 12) and the Silnica River downstream (sampling point no. 11), the $\mathrm{pH}$ increased from 3.3 to 6.9 and the concentrations of $\mathrm{SO}_{4}{ }^{2-}$ and $\mathrm{Cu}$ dropped from 423 to $134 \mu \mathrm{g} / \mathrm{L}$ and from 878 to $86 \mu \mathrm{g} / \mathrm{L}$, respectively. The same downward trend was also noted for the REE (from 127.5 to $5.6 \mu \mathrm{g} / \mathrm{L}$ ), $\mathrm{Al}$ (from 18.9 to $0.44 \mathrm{mg} / \mathrm{L}$ ), $\mathrm{Fe}$ (from 10.6 to $0.4 \mathrm{mg} / \mathrm{L}$ ), and As (from 25.3 to $2.2 \mu \mathrm{g} / \mathrm{L}$ ). Another significant issue is the presence of exposed and unexposed piles derived from former mining operations, especially from the Podwiśniówka quarry, which still contribute to elevated metal(loid) concentrations (of which arsenic is of greatest concern) in intermittent strongly acidic pools.

Unfavorable localization of the Wiśniówka mining area, i.e., a dismembered mountain range reaching $100 \mathrm{~m}$ in relative elevation and the proximity of villages, makes it difficult to utilize abiotic or biotic remediation strategies (e.g., Banks et al. 1997; Plumlee and Logsdon 1999; Coulton et al. 2003; Johnson and Hallberg 2005; Runkel et al. 2009). In addition, the lack of flat areas for construction of large biotic aerobic ponds or wetlands and (an) aerobic plots has forced the mining company to use other options of AMD water remediation, including anoxic limestone drains, impermeable barriers to potential acid seeps, lime addition, and revegetation of some tailings piles.

No action has in turn been taken against the Podwiśniówka acid pit lake. This part of the quarry is naturally watertight and to date has not posed a threat to the adjacent river systems or suspended local aquifers as indicated by the previous studies of farmer's well waters (Migaszewski et al. 2014) and current monitoring. The only solution is to isolate the major part of the reactive pyrite mineralization zone by covering it with clays and soil, grassing over and planting with trees. Although currently the Podwiśniówka acid pit lake does not jeopardize the local underground water system, this site needs constant monitoring to evaluate its potential impact on the neighboring environment especially during long periods of rainfalls.

\section{Conclusions}

The following conclusions can be drawn from the hydrogeochemical data derived from this study:
1. Different shale-normalized REE concentration patterns from the Podwiśniówka acid pit lake (roof-shaped with $\mathrm{Gd}, \mathrm{Eu}$ and $\mathrm{Tb}$ positive anomalies) and the Wiśniówka acid pit sump (step-shaped with a strong predominance of HREE $_{\text {NASC }}$ over LREE $_{\text {NASC }}$ ) can be used along with some metal(loid)s as geochemical signatures of mining operations and their potential influence on the environment.

2. Due to fractionation of the REE during weathering, leaching of dissolved and suspended fractions, and geochemical interactions in the acid water bodies, the individual REE show greater affinities to Mn, HREE to Fe and $\mathrm{SO}_{4}{ }^{2-}$, and $\mathrm{La}$ and $\mathrm{Ce}$ to $\mathrm{Al}$.

3. The chemistry of some acid pools provides information on unexposed pyrite occurrences within the intensively folded and faulted Wiśniówka massif. This may give a clue to the future mining operations in a tectonically affected area.

4. The acid water bodies of the Wiśniówka mining area currently show a minor impact on the surface and underground water systems. However, the results derived from this study also indicate that the Podwiśniówka acid lake and acid pools should be protected against accidental surficial runoff or leachate by constructing earth barriers or using naturally isolated depressions. Moreover, these "hot spots" needs constant monitoring to evaluate their impact on the neighboring environment.

The obtained data enable mine planners to better understand, anticipate, and mitigate potential environmental hazards. These have also been useful for working out realistic estimates and plans for quartzite extraction and processing, as well as for developing remediation and monitoring programs.

Acknowledgments The authors are grateful to Mr. Leszek Giro of the Polish Geological Institute-National Research Institute for taking SEM images. We would also like to extend our thanks to three anonymous reviewers whose remarks considerably improved the quality of our manuscript. This study was supported by the National Science Center, a research grant no. 2015/17/B/ST10/02119.

Open Access This article is distributed under the terms of the Creative Commons Attribution 4.0 International License (http:// creativecommons.org/licenses/by/4.0/), which permits unrestricted use, distribution, and reproduction in any medium, provided you give appropriate credit to the original author(s) and the source, provide a link to the Creative Commons license, and indicate if changes were made.

\section{References}

Acero P, Ayora C, Torrentó C, Nieto J-M (2006) The behavior of trace elements during schwertmannite precipitation and subsequent transformation into goethite and jarosite. Geochim Cosmochim Acta 70: 4130-4139

Aguilar J, Dorronsoro C, Fernández E, Fernández ZJ, Garcia I, Martin F, Simón M (2004) Soil pollution by a pyrite mine spill in Spain: evolution with time. Environ Pollut 132:395-401 
Banks D, Younger PL, Arnesen RT, Iversen ER, Banks SB (1997) Minewater chemistry: the good, the bad and the ugly. Environ Geol 32(3): $157-174$

Bau M, Dulski P (1996) Distribution of yttrium and rare-earth elements in the Penge and Kuruman iron-formations, Transvaal Supergroup, South Africa. Precambrian Res 79:37-55

Bednar AJ, Garbarino JR, Ranville JF, Wildeman TR (2005) Effects of iron on arsenic speciation and redox chemistry in acid mine water. J Geochem Explor 85:55-62

Bingham JM, Schwertmann U, Carlson L, Murad E (1990) A poorly crystallized oxyhydroxysulfate of iron formed by bacterial oxidation of $\mathrm{Fe}$ (II) in acid mine waters. Geochim Cosmochim Acta 54:2743-2758

Bingham JM, Schwertmann U, Carlson L (1992) Mineralogy of precipitates formed by biogeochemical oxidation of $\mathrm{Fe}$ (II) in mine drainage. In: Skinner HCW, Fitzpatrick RW (eds) Biomineralization process of iron and manganese - modern and ancient environments. Cremlingen-Destedt Germany, Catena Verlag, pp. 219-232

Borrego J, Carro N, López-González N, de la Rosa J, Grande JA, Gómez T, de la Torre ML (2012) Effect of acid mine drainage on dissolved rare earth elements geochemistry along a fluvial-estuarine system: the Tinto-Odiel estuary (S.W. Spain. Hydrol Res 43:262-274

Bozau E, Leblanc M, Seidel JL, Stärk H-J (2004) Light rare earth elements enrichment in an acidic mine lake (Lusatia, Germany. Appl Geochem 19:261-271

Cánovas CR, Hubbard CG, Olías M, Nieto JM, Black S, Coleman ML (2008) Hydrochemical variations and contaminant load in the Rio Tinto (Spain) during flood events. J Hydrol 350:25-40

Coppin F, Berger G, Bauer A, Castet S, Loubet M (2002) Sorption of lanthanides on smectite and kaolinite. Chem Geol 182:57-68

Coulton R, Bullen C, Dolan J, Hallet C, Wright J, Marsden C (2003) Wheal Jane mine water active treatment plant-design, construction and operation. Land Contam Reclam 11:245-252

Courtin-Nomade A, Grosbois C, Bril H, Roussel C (2005) Spatial variability of arsenic in some iron-rich deposits generated by acid mine drainage. Appl Geochem 20:383-396

Druschel GK, Baker BJ, Gihring TM, Banfield JF (2004) Acid mine drainage biogeochemistry at Iron Mountain, California. Geochem T 5(2):13-32

España JS, Pamo EL, Pastor ES, Ercilla MD (2008) The acidic mine pit lakes of the Iberian Pyrite Belt: an approach to their physical limnology and hydrogeochemistry. Appl Geochem 23:1260-1287

Gammons CH, Wood SA, Jonas JP, Madison JP (2003) Geochemistry of rare earth elements and uranium in the acidic Berkeley Pit lake, Butte, Montana. Chem Geol 198:269-288

Gammons CH, Wood SA, Pedrozo F, Varekamp JC, Nelson BJ, Shope CL, Baffico G (2005) Hydrogeochemistry and rare earth element behavior in a volcanically acidified watershed in Patagonia, Argentina. Chem Geol 222:249-267

Garrels RM, Thompson ME (1960) Oxidation of pyrite in ferric sulfate solutions. Am J Sci 258:57-67

German CR, Hollday BP, Elderfield H (1991) Redox cycling of rare earth elements in the suboxic zone of the Black Sea. Geochim Cosmochim Acta 55:3553-3558

Giloteaux L, Duran R, Casiot C, Bruneel O, Elbaz-Poulichet F, GoňiUrriza M (2012) Three-year survey of sulfate-reducing bacteria community structure in Carnoulés acid mine drainage (France), highly contaminated by arsenic. FEMS Microb Ecol 83:724-737

Gimeno Serrano MJ, Auqué LF, Nordstrom DK (2000) REE speciation in low-temperature acidic waters and the competitive effects of aluminum. Chem Geol 165:167-180

Grawunder A, Merten D, Büchel G (2014) Origin of middle rare earth element enrichment in acid mine drainage-impacted areas. Environ Sci Pollut Res 21:6812-6823

Gromet LP, Dymek RF, Haskin LA, Korotev RL (1984) The "North American shale composite"; its compilation, major and trace element characteristics. Geochim Cosmochim Acta 48:2469-2482
Hannigan RE, Sholkovitz ER (2001) The development of middle rare earth element enrichments in freshwaters: weathering of phosphate minerals. Chem Geol 175:495-508

Haskin LA, Wildeman TR, Haskin MA (1968) An accurate procedure for the determination of the rare earths by neutron activation. J Radioanal Nucl Ch 1:337-348

Hudson-Edwards KA, Schell C, Macklin MG (1999) Mineralogy and geochemistry of alluvium contaminated by metal mining in the Rio Tinto area, southwest Spain. Appl Geochem 14:1015-1030

Ingri J, Widerlund A, Land M, Gustafsson Ö, Andersson P, Öhlander B (2000) Temporal variations in the fractionation of the rare earth elements in a boreal river; the role of colloidal particles. Chem Geol 166:23-45

ISO/IEC:17025 (2005) General requirements concerning competence of research and accredited laboratories. PKN. Warszawa

Jaworowski K, Sikorska M (2006) Łysogory Unit (Central Poland) versus East European Craton-application of sedimentological data from Cambrian siliciclastic association. Geol Quart 50:77-88

Johannesson KH, Lyons WB (1995) Rare-earth element geochemistry of Colour Lake, an acidic freshwater lake on Axel Heiberg Island, Northwest Territories, Canada. Chem Geol 119:209-223

Johannesson KH, Zhou XP (1999) Origin of middle rare earth element enrichments in acid waters of a Canadian High Arctic lake. Geochim Cosmochim Acta 63:153-165

Johnson DB, Hallberg KB (2005) Acid mine drainage remediation options: a review. Sci Total Environ 338:3-14

Knappe A, Möller P, Dulski P, Pekdeger A (2005) Positive gadolinium anomaly in surface water and ground water of the urban area Berlin, Germany. Chemie der Erde Geochem 65:167-189

Kolker A, Nordstrom, DK (2001) Occurrence and micro-distribution of arsenic in pyrite. U.S. Geol Surv website (http://wwwbrr.cr.usgs.gov /Arsenic/)

Konon A (2008) Tectonic subdivision of Poland: Holy Cross Mountains and adjacent areas. Prz Geol 56:921-926 (in Polish)

Kulaksiz S, Bau M (2011) Rare earth elements in the Rhine River, Germany: first case of anthropogenic lanthanum as a dissolved microcontaminant in the hydrosphere. Environ Int 37:973-979

Leybourne MI, Johannesson KH (2008) Rare earth elements (REE) and yttrium in stream waters, stream sediments, and $\mathrm{Fe}-\mathrm{Mn}$ oxyhydroxides: fractionation, speciation, and controls over REE + Y patterns in the surface environment. Geochim Cosmochim Acta 72:5962-5983

Leybourne MI, Goodfellow WD, Boyle DR, Hall GM (2000) Rapid development of negative $\mathrm{Ce}$ anomalies in surface waters and contrasting REE patterns in groundwaters associated with $\mathrm{Zn}-\mathrm{Pb}$ massive sulphide deposits. Appl Geochem 15:695-723

Martínez- Martínez S, Acosta JA, Faz Cano S, Carmona DM, Zornoza R, Cerda C (2013) Assessment of the lead and zinc contents in natural soils and tailing ponds from the Cartagena-La Unión mining district, SE Spain. J Geochem Explor 124:166-175

McLennan SM (1994) Rare earth element geochemistry and the "tetrad" effect. Geochim Cosmochim Acta 58:2025-2033

Merten D, Geletneky J, Bergmann H, Haferburg G, Kothe E, Büchel G (2005) Rare earth element patterns: a tool for understanding processes in remediation of acid mine drainage. Chem Erde 65:97-114

Merten D, Grawunder A, Lonschinski M, Lorenz C, Büchel G (2007) Rare earth element patterns related to bioremediation processes in a site influenced by acid mine drainage. In: Cidu R, Frau F (eds) Water in mining environments, intern mine water Assoc Symp. Cagliary, Italy, pp. 233-237

Miekeley N, Couthino de Jesus H, Porto da Silveira CL (1992) Rare earth elements in groundwater from the Osamu Utsumi mine and Morro do Ferro analogue study sites, Plcos de Caldas, Brazil. J Geochem Explor 45:365-387 
Migaszewski ZM, Gałuszka A (2010) Xenotime from the Podwiśniówka mine pit, Holy Cross Mountains (South-Central Poland). Mineralogia 1-2:1-7

Migaszewski ZM, Gałuszka A (2015) The characteristics, occurrence and geochemical behavior of rare earth elements in the environment: a review. Crit Rev Env Sci Tech 45:429-471

Migaszewski ZM, Gałuszka A (2016) The use of gadolinium and europium concentrations as contaminant tracers in the Nida River watershed in south-central Poland. Geol Quart 60(1):65-74

Migaszewski ZM, Gałuszka A, Pasławski P, Starnawska E (2007a) An influence of pyrite oxidation on generation of unique acid pit water: a case study, Podwiśniówka quarry, Holy Cross Mountains (|southCentral Poland). Pol J Environ S 16(3):407-421

Migaszewski ZM, Starnawska E, Gałuszka A (2007b) Gorceixite from the Upper Cambrian rocks of the Podwiśniówka mine pit, Holy Cross Mountains (south-central Poland). Miner Pol 38(2):171-184

Migaszewski ZM, Gałuszka A, Hałas S, Dołegowska S, Dąbek J, Budzyk I, Starnawska E (2008) Geochemistry and stable sulfur and oxygen isotope ratios of the Podwiśniówka pit pond water generated by acid mine drainage (Holy Cross Mountains, south-central Poland. Appl Geochem 23:3620-3634

Migaszewski ZM, Gałuszka A, Hałas S, Dąbek J, Dołegowska S, Budzyk I, Starnawska E, Michalik A (2009) Chemical and isotopic variations in the Wiśniówka Mała mine pit water, Holy Cross Mountains (South-Central Poland. Environ Geol 57:29-40

Migaszewski ZM, Gałuszka A, Michalik A, Dołegowska S, Migaszewski A, Hałas S, Trembaczowski A (2013) The use of stable sulfur, oxygen and hydrogen isotope ratios as geochemical tracers of sulfates in the Podwiśniówka acid drainage area (South-Central Poland. Aquat Geochem 19:261-280

Migaszewski ZM, Gałuszka A, Migaszewski A (2014) The study of rare earth elements in farmer's well waters of the Podwiśniówka acid mine drainage area (south-central Poland. Environ Monit Assess 186:1609-1622

Migaszewski ZM, Gałuszka A, Dołęgowska S, Hałas S, Krzciuk K, Gebus B (2015) Assessing the impact of Serwis mine tailings site on farmer's wells using element and isotope signatures (Holy Cross Mountains, south-central Poland. Environ Earth Sci 74:629-647

Mighanetara K, Braungardt CB, Rieuwerts JS, Azizi F (2009) Contaminant fluxes from point and diffuse sources from abandoned mines in the River Tamar catchment, UK. J Geochem Explor 100: $116-62124$

Monterroso C, Macías F (1998) Drainage waters affected by pyrite oxidation in a coal mine in Galicia (NW Spain): composition and mineral stability. Sci Total Environ 216:121-132

Moses CO, Nordstrom DK, Herman JS, Mills AL (1987) Aqueous pyrite oxidation by dissolved oxygen and by ferric iron. Geochim Cosmochim Acta 51:1561-1571

NORDIC INNOVATION CENTRE (2007) Uncertainty from sampling. A Nordtest Handbook for Sampling Planners on Sampling Quality Assuarance and Uncertainty Estimation

Nordstrom DK (2011) Hydrogeochemical processes governing the origin, transport and fate of major and trace elements from mine wastes and mineralized rock to surface waters. Appl Geochem 26:17771791

Nordstrom DK, Alpers CN (1999a) Geochemistry of acid mine waters. In: Plumlee GS, Logsdon MJ (Eds) The environmental geochemistry of mineral deposits, part a. Processes, techniques, and health issues. Soc econ geologists. Rev in Econ Geol 6A:133-160

Nordstrom DK, Alpers CN (1999b) Negative pH, efflorescent mineralogy, and consequences for environmental restoration at the Iron Mountain Superfund site, California. Proceedings Natl. Acad Sci 96:3455-3462

Olías M, Caánovas CR, Nieto JM, Sarmiento AM (2006) Evaluation of the dissolved contaminant load transported by the Tinto and Odiel rivers (South West Spain. Appl Geochem 21:1733-1749
Orłowski S (1975) Cambrian and Upper Precambrian lithostratigraphic units in the Holy Cross Mts. Acta Geol Pol 25:431-448

Pérez-López R, Gelgado J, Nieto JM, Márquez-García B (2010) Rare earth element geochemistry of sulphide weathering in the São Domingos mine area (Iberian Pyrite Belt): a proxy for fluid-rock interaction and ancient mining pollution. Chem Geol 276:29-40

Plumlee GS, Logsdon MJ (1999) An earth-system science toolkit for environmentally friendly mineral resource development. In: Plumlee GS, Logsdon MJ (Eds) The environmental geochemistry of mineral deposits, part A. Processes, techniques, and health issues. Soc econ geologists. Rev in Econ Geol 6A:1-27

Plumlee GS, Smith KS, Montour MR, Ficklin WH, Mosier EL (1999) Geologic controls on the composition of natural waters and mine waters draining diverse mineral-deposits types. In: Filipek LH, Plumlee GS (Eds) The environmental geochemistry of mineral deposits. Soc econ geologists. Rev in Econ Geol 6B:373-407

Protano G, Riccobono F (2002) High contents of rare earth elements (REEs) in stream waters of a $\mathrm{Cu}-\mathrm{Pb}-\mathrm{Zn}$ mining area. Environ Pollut 117:499-514

Reichenbach I (1994) Black shale as an environmental hazard; a review of black shales in Canada. Geol Surv of Canada Open-File 2697

Ribbe PH (1982) Review in Mineralogy, vol. 1: Sulfide mineralogy, Min Soc America, $284 \mathrm{p}$

Romero FM, Prol-Ledesma RM, Canet C, Alvares LN, Pérez-Vázquez R (2010) Acid drainage at the inactive Santa Lucia mine, western Cuba: natural attenuation of arsenic, barium and lead, and geochemical behavior of rare earth elements. Appl Geochem 25:716-727

Runkel RL, Bencala KE, Kimball BA, Walton-Day K, Verplanck PL (2009) A comparison of pre- and post-remediation water quality, Mineral Creek, Colorado. Hydrol Process 23:3319-3333

Schwertmann U, Bingham JM, Murad E (1995) The first occurrence of schwertmannite in a natural stream environment. European $\mathrm{J}$ Mineral 7:547-552

Sholkovitz ER (1995) The aquatic chemistry of rare earth elements in rivers and estuaries. Aquat Geochem 1:1-34

Smedley PL, Kinniburgh DG (2002) A review of the source, behaviour and distribution of arsenic in natural waters. Appl Geochem 17:517568

The Regulation of the Minister of the Environment on the conditions that need to be met when releasing wastewater to surface waters or soils, and on the substances particularly harmful to water environment (2014) J Acts Dziennik Ustaw:1800

Verplanck PL, Antweiler RC, Nordstrom DK, Taylor HE (2001) Standard reference water samples for rare earth element determinations. Appl Geochem 16:231-244

Verplanck PL, Nordstrom DK, Taylor HE, Kimball BA (2004) Rare earth element partitioning between hydrous ferric oxides and acid mine water during iron oxidation. Appl Geochem 19:1339-1354

Winterbourn MJ, McDiffett WF, Eppley SJ (2000) Aluminium and iron burdens of aquatic biota in New Zealand streams contaminated by acid mine drainage: effects of trophic level. Sci Total Environ 254: $45-54$

Worrall F, Pearson DG (2001) Water-rock interaction in an acidic mine discharge as indicated by rare earth element patterns. Geochim Cosmochim Acta 65:3027-3040

Younger PL, Coulton RH, Froggatt EC (2005) The contribution of science to risk-based decision-making: lessons from the development of full-scale treatment measures for acidic mine waters at Wheal Jane, UK. Sci Total Environ 338:137-154

Zhao F, Cong Z, Sun H, Ren D (2007) The geochemistry of rare earth elements (REE) in acid mine drainage from the Sitai coal mine, Shanxi Province, North China. Int J Coal Geol 70:184-192

Żylińska A, Szczepanik Z, Salwa S (2006) Cambrian of the Holy Cross Mountains, Poland: biostratigraphy of the Wiśniówka Hill succession. Acta Geol Pol 56(4):443-461 